\title{
Two-Dimensional Mathematical Treatment of Small-Angle X-Ray Scattering from a System of Alternating Lamellar Phases with Orientation Distribution
}

\author{
Masaru MATSUO* and Chikako KITAYAMA \\ Department of Clothing Science, Faculty of Home Economics, \\ Nara Women's University, Nara 630, Japan
}

(Received June 11, 1984)

\begin{abstract}
The small angle X-ray scattering from alternating lamellar phases may formulated on the basis of two concepts. The one is that the scattering is due to positive and negative density deviations from the average density of the system and the other is that the scattering depends upon the excess electron density between the two phases. In order to consider scattering from real systems, the mathematical treatment is carried out for two dimensional lamellar assemblies with orientational distribution. The scattering pattern calculated on the former concept is in good agreement with ones which have been observed experimentally. By contrast, the pattern calculated on the latter concept has many scattering maxima which have never been observed. The origin of this discrepancy for the latter concept has been derived from its scattering equation.

KEY WORDS Small Angle X-Ray Scattering / Alternating Lamellar Phases / Average Density / Excess Electron Density / Two Dimensional Lamellar Assemblies /
\end{abstract}

There are some current theories ${ }^{1-3}$ for the small angel X-ray scattering (SAXS) from alternating lamellar phases. They are entirely formulated on the basis of the concept that the scattering is due to positive and negative density deviations from the average density of the system. This concept differs from the approach of Hosemann $n^{4,5}$ and is more suitable for analyzing the scattering from real systems. Hosemann's approach is based on the assumption that the scattering depends upon the excess electron density between the crystalline and amorphous regions. Namely, his approach indicates in principle that a given assembly consisting of several pairs of crystalline and amorphous regions is floating in an amorphous infinite medium. Blundell ${ }^{6,7}$ extended Hosemann's approach ${ }^{4}$ to a general one dimensional model where the density of a finite transition zone between crystalline and amorphous phases changes linearly and where the thickness of the crystalline and amorphous domains fluctuates according to independent Gaussian distributions. However, a real system consists of many assemblies and it must be considered that a given assembly is surrounded by an infinite medium whose density is equivalent to the average density of the given system. Our previous paper ${ }^{3}$ was concerned with a theoretical formulation in which the scattering was due to positive and negative density deviations from the average density of the system. Using this approach, it turns out that the scattered intensity could be represented as a function of the volume crystallinity (for a crystalline polymer) or the volume fraction (for a block or graft copolymer) if these polymers consist of alternating lamellar domains. The resulting equations of SAXS intensity satisfies

\footnotetext{
* To whom all correspondence should be addressed.
} 
Babinet's theorem for the volume crystallinity or the volume fraction when there is no fluctuation in the thickness of the alternating lamellar phases.

However, in our previous paper, ${ }^{3}$ the length in crystalline lamellae and the orientational fluctuation of lamellar assemblies were not introduced in the theoretical calculation. Such a treatment is not sufficient to explain the scattering from the real system. Accordingly, the focus of this paper is concentrated on a general theoretical treatment in which an assembly consisting of several lamellae with finite size has orientational fluctuation with respect to a particular direction and the given assembly is surrounded by an infinite medium whose density is equivalent to the average density of the given system. Numerical calculations were carried out for the azimuthal dependence of the scattered intensity and the results calculated were compared with those calculated by Blundell's model. ${ }^{6,7}$

\section{THEORY}

The coordinate system is given in the case when a film composes of large lamellar crystals stacked on top of one another to form a periodic crystalline-amorphous structure parallel to the plane of the film. This arrangement has been confirmed by SAXS patterns for the dry gel films produced by gelation/ crystallization from semidilute solutions, ${ }^{8-10}$ and for styrene-isoprene block copolymer films cast from toluene solutions. ${ }^{11}$ Figure 1 shows the coordinate to calculate the scattered intensity from the above system. In this model system, the $X_{3}$ axis corresponds to the film normal direction and the $X_{1}$ axis is parallel to the direction of an incident beam whose unit vector is defined as $s_{0}$. The intensity distribution is observed as a function of the scattering angle $2 \theta$ and the azimuthal angle $\mu$, and $\boldsymbol{s}^{\prime}$ is a unit vector along the scattered X-ray beam. In order to simplify the calculations, it is assumed that the crystalline lamellae are rec-

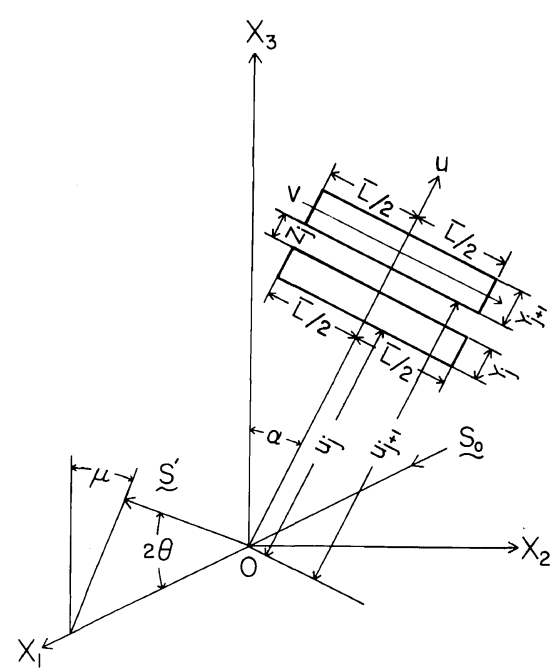

Figure 1. The models used for the theoretical analysis of the SAXS intensity distribution.

tangular in cross-section and situated in the plane $0-X_{2} X_{3}$. The center of gravity of each crystalline lamellae is in the direction of the $u$ axis. The dimensions of the $j$-th lamellae are represented as the $\bar{L}$ and $\bar{Y}_{j}$. The $u$ axis is fixed on the $0-X_{2} X_{3}$ plane. The size of lemella in the $X_{1}$ direction is neglected, since this affects only on the magnitude of the scattered intensity but is independent of the intensity distribution. In this model system, it is assumed that all the lamellae have same length $\bar{L}$. The distance between the $j$-th and th $(j+1)$-th lamellae is defined as $Z_{j}$ and $\alpha$ denotes the orientational angle between $X_{3}$ and $u$ axes. In order to represent the variation of density along the $u$ axis, two schematic diagrams are proposed. Incidentally, for mathematical simplicity, the variation along the $v$ axis, which is parallel in the direction of lamellar length, is neglected.

Figure 2 shows schematic diagrams to represent that the density variation of two phases is specified by positive and negative deviations from the average density of the system, ${ }^{3}$ in which (a) the variation is specified by an arbitrary function and (b) a trapezoidal function. This type of diagram is named as A-type. The lengths $Y_{j}$ and $Z_{j}$ are those 


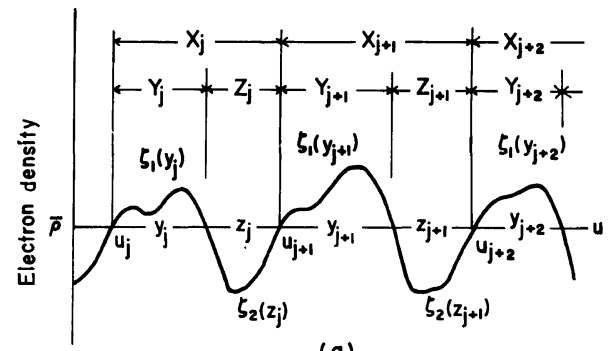

(a)

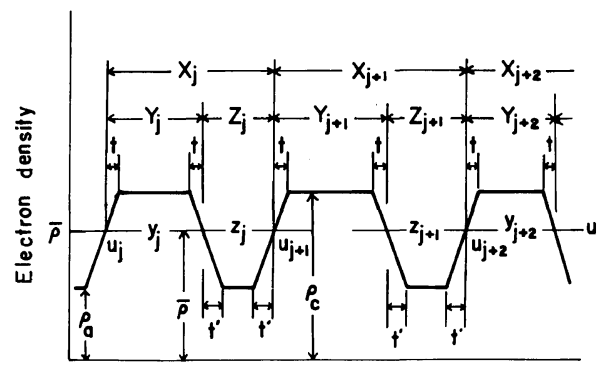

(b)

Figure 2. A-type models for representing the SAXS intensity distribution, in which the density variation between the two phases is specified by positive and negative deviations from the average of the system. (a) Variation of electron density specified by the arbitrary functions $\zeta_{1}(y)$ and $\zeta_{2}(z)$; (b) Trapezium density profile corresponding to $\zeta_{1}(y)$ and $\zeta_{2}(z)$.

of the representative phases and $X_{j}$ is the total length (the repeat distance) of the $j$-th pair. The lengths $t$ and $t^{\prime}$ of the transition zones are assumed to be constant whereas the lengths $Y_{j}$ and $Z_{j}$ are assumed to fluctuate independently from the average values $\bar{Y}$ and $\bar{Z}$.

Figure 3 shows schematic diagrams proposed by Blundell, ${ }^{6}$ in which (a) the density variation is specified by the excess electron density denoting the density difference between alternating lamellar phases and (b) shows a trapezoidal density variation corresponding to (a). This type of diagram is named as B-type. In B-type models, the density of the phase with the length $Z_{j}$ is thought to be zero. The length $t$ corresponds to the transition zone of the two phases of $Y_{j}$ and $Z_{j}$.

In our previous work, ${ }^{3}$ the treatment was formulated in the case when infinitesimally thin lamellae in length are arranged in the

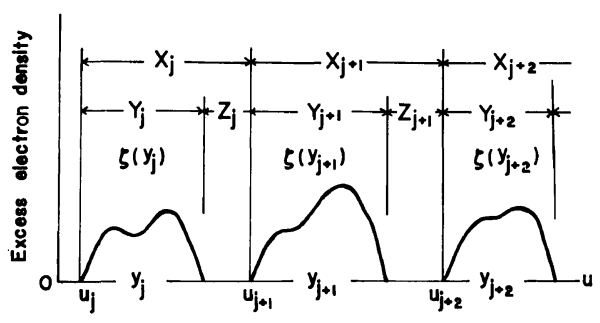

(a)

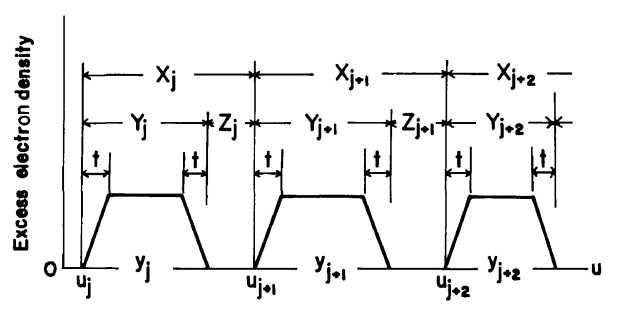

(b)

Figure 3. B-type models for calculating the SAXS intensity distribution. (a) Variation of excess electron density specified by an arbitrary functions $\zeta(y)$; (b) Trapezium density profile corresponding to $\zeta(y)$.

direction of $X_{3}$ axis as $\alpha=0^{\circ}$ in Figure 1 . Namely, it was assumed that the intensity is based on the scattering from the density variation along the $X_{3}$ axis. Therefore, as can be understood, our current theory is more realistic to represent the scattered intensity.

\section{General Model}

Considering the geometrical arrangements described in Figures 1 and 2, the scattering amplitude from the $N$ pairs of phases is given by

$a(s)$

$$
\begin{aligned}
& =\int_{-\bar{L} / 2}^{\bar{L} / 2} \int_{u_{1}}^{u_{N}+1} \rho(u) \exp [-2 \pi i(b u-a v)] \mathrm{d} u \mathrm{~d} v \\
& =\sum_{j=1}^{N} \int_{-\bar{L} / 2}^{\bar{L} / 2} \int_{u_{j}}^{u_{j+1}} \rho(u) \\
& \quad \quad \quad \exp [-2 \pi i(b u-a v)] \mathrm{d} u \mathrm{~d} v
\end{aligned}
$$

where $a$ and $b$ in eq 1 are given as a function of $s(=\sin 2 \theta / \lambda)$ as follows: 


$$
a=s \sin (\alpha-\mu)
$$

and

$$
b=s \cos (\alpha-\mu)
$$

are the Bragg and azimuthal angles, and $\lambda$ is the X-ray wavelength in the medium. $\rho(u)$ in eq 1 denotes the density distribution in the direction of $u$ axis and can be rewritten as $\zeta_{1}(u)$ $\left(0 \leqq y_{j} \leqq Y_{j}\right)$ and $\zeta_{2}(u)\left(0 \leqq z_{j} \leqq Z_{j}\right)$. Then

$N$ is the number of pairs of domains. $\theta$ and $\mu$

$$
\begin{aligned}
a(s)= & \sum_{j=1}^{N} \int_{-\bar{L} / 2}^{\bar{L} / 2} \int_{0}^{Y_{j}} \zeta_{1}\left(y_{j}\right) \exp \left[-2 \pi i\left\{b\left(u_{j}+y_{j}\right)-a v\right\}\right] \mathrm{d} y_{j} \mathrm{~d} v \\
& +\sum_{j=1}^{N} \int_{-\bar{L} / 2}^{\bar{L} / 2} \int_{0}^{z_{j}} \zeta_{2}\left(z_{j}\right) \exp \left[-2 \pi i\left\{b\left(u_{j}+Y_{j}+z_{j}\right)-a v\right\}\right] \mathrm{d} z_{j} \mathrm{~d} v \\
= & \sum_{j=1}^{N} \exp \left[-2 \pi i b u_{j}\right] f_{j}+\sum_{j=1}^{N} \exp \left[-2 \pi i b\left(u_{j}+Y_{j}\right)\right] g_{j}
\end{aligned}
$$

where

$$
\begin{aligned}
f_{j} & =\int_{-\bar{L} / 2}^{\bar{L} / 2} \int_{0}^{Y_{j}} \zeta_{1}\left(y_{j}\right) \exp \left[-2 \pi i\left(b y_{j}-a v\right)\right] \mathrm{d} y_{y} d v \\
& =\frac{\sin (\pi a \bar{L})}{\pi a} \int_{0}^{Y_{j}} \zeta_{1}\left(y_{j}\right) \exp \left[-2 \pi i b y_{j}\right] \mathrm{d} y_{j} \\
g_{j} & =\int_{-\bar{L} / 2}^{\bar{L} / 2} \int_{0}^{Z_{j}} \zeta_{2}\left(z_{j}\right) \exp \left[-2 \pi i\left(b z_{j}-a v\right)\right] \mathrm{d} z_{j} \mathrm{~d} v \\
& =\frac{\sin (\pi a \bar{L})}{\pi a} \int_{0}^{Z_{j}} \zeta_{2}\left(z_{j}\right) \exp \left[-2 \pi i b z_{j}\right] \mathrm{d} z_{j}
\end{aligned}
$$

Considering the orientation distribution func- to the $X_{3}$ axis, the average scattered intensity is tion $p(\alpha)$ of the lamellar assembly with respect given by

$$
\begin{aligned}
I(s) & =\int_{0}^{2 \pi} p(\alpha) a(s) a^{*}(s) \mathrm{d} \alpha / \int_{0}^{2 \pi} p(\alpha) \mathrm{d} \alpha \\
& =\left(I_{1}+I_{2}+I_{3}+I_{4}\right) / \int_{0}^{2 \pi} p(\alpha) \mathrm{d} \alpha
\end{aligned}
$$

where

$$
\begin{aligned}
& I_{1}=\sum_{j=1}^{N} \sum_{k=1}^{N} \int_{0}^{2 \pi} p(\alpha) \exp \left\{-2 \pi i b\left(u_{j}-u_{k}\right)\right\} f_{j} f_{k}^{*} \mathrm{~d} \alpha \\
& I_{2}=\sum_{j=1}^{N} \sum_{k=1}^{N} \int_{0}^{2 \pi} p(\alpha) \exp \left\{-2 \pi i b\left(u_{j}+Y_{j}-u_{k}-Y_{k}\right)\right\} g_{j} g_{k}^{*} \mathrm{~d} \alpha \\
& I_{3}=\sum_{j=1}^{N} \sum_{k=1}^{N} \int_{0}^{2 \pi} p(\alpha) \exp \left\{-2 \pi i b\left(u_{j}-u_{k}-Y_{k}\right)\right\} f_{j} g_{k}^{*} \mathrm{~d} \alpha
\end{aligned}
$$




$$
I_{4}=\sum_{j=1}^{N} \sum_{k=1}^{N} \int_{0}^{2 \pi} p(\alpha) \exp \left\{-2 \pi i b\left(u_{j}+Y_{j}-u_{k}\right)\right\} g_{j} f_{k}^{*} \mathrm{~d} \alpha
$$

$a^{*}(s), f^{*}$, and $g^{*}$ denote complex conjugates of $a(s), f$, and $g$, respectively. The scattered intensity corresponds to the real parts of eq $7 \mathrm{a}-$ $7 \mathrm{~d}$ and the real part of $I_{3}$ is equivalent to that of $I_{4}$. Incidentally, the sum of the each imaginary part of eq $7 \mathrm{a}-7 \mathrm{~d}$ is zero, if the lamellae possess a center of symmetry.

Following Blundell, ${ }^{6}$ the scattered intensity may be normalized as $a(s) a^{*}(s) / N \bar{X} \bar{L}$, where $\bar{X}$ $(=\bar{Y}+\bar{Z})$ denotes the mean periodic distance and therefore $N \bar{X} \bar{L}$ corresponds to the area of the assembly of lamellae. Thus, the total scattered intensity per unit area of scattering structure will be given by

$$
\frac{I}{N \bar{L} \bar{X}}=\frac{1}{N \bar{L} \bar{X}}\left(I_{1}+I_{2}+2 I_{3}\right) / \int_{0}^{2 \pi} p(\alpha) \mathrm{d} \alpha
$$

By using a similar mathematical treatment by Blundell, ${ }^{6}$ the $I_{1}, I_{2}$, and $I_{3}$ terms may be represented as a similar form proposed by him, which are given by

$$
\begin{gathered}
\frac{I_{1}}{N \bar{L} \bar{X}}=I_{1}^{\mathrm{B}}-I_{1}^{\mathrm{C}} \\
I_{1}^{\mathrm{B}}=\int_{0}^{2 \pi} p(\alpha) \operatorname{Re}\left\{\frac{1}{\bar{X} \bar{L}}\left[J_{1}+\frac{2 G_{y} \gamma_{y} F_{z}}{1-F_{y} F_{z}}\right]\right\} \mathrm{d} \alpha \\
I_{1}^{\mathrm{C}}=\int_{0}^{2 \pi} p(\alpha) \operatorname{Re}\left\{\frac{2 G_{y} \gamma_{y} F_{z}\left[1-\left(F_{y} F_{z}\right)^{N}\right]}{N \bar{X} \bar{L}\left(1-F_{y} F_{z}\right)^{2}}\right\} \mathrm{d} \alpha \\
I_{2}^{\mathrm{B}}=\int_{0}^{2 \pi} p(\alpha) \operatorname{Re}\left\{\frac{1}{\bar{X} \bar{L}}\left[J_{2}+\frac{2 A_{z} B_{z} F_{y}}{1-F_{y} F_{z}}\right]\right\} \mathrm{d} \alpha \\
I_{2}^{\mathrm{C}}=\int_{0}^{2 \pi} p(\alpha) \operatorname{Re}\left\{\frac{2 A_{z} B_{z} F_{y}\left[1-\left(F_{y} F_{z}\right)^{N}\right]}{N \bar{X} \bar{L}\left(1-F_{y} F_{z}\right)^{2}}\right\} \mathrm{d} \alpha \\
I_{3}^{\mathrm{B}}=\int_{0}^{2 \pi} p(\alpha) \operatorname{Re}\left\{\frac{1}{\left.\bar{X} \bar{L}\left[\frac{2\left(G_{y} B_{z}+A_{z} \gamma_{y}\right)}{1-F_{y} F_{z}}\right]\right\} \mathrm{d} \alpha}\right. \\
I_{3}^{\mathrm{C}}=\int_{0}^{2 \pi} p(\alpha) \operatorname{Re}\left\{\frac{2\left(G_{y} B_{z} F_{y} F_{z}+\gamma_{y} A_{z}\right)\left[1-\left(F_{y} F_{z}\right)^{N}\right]}{N \bar{X} \bar{L}\left(1-F_{y} F_{z}\right)^{2}}\right\} \mathrm{d} \alpha
\end{gathered}
$$

where

$$
\begin{array}{rlrl}
J_{1} & =\int_{-\infty}^{\infty}\left|f_{j}\right|^{2} H\left(Y_{j}\right) \mathrm{d} Y_{j} & (15 \mathrm{a}) & G_{y}=\int_{-\infty}^{\infty} f_{j}^{*} H\left(Y_{j}\right) \exp \left(-2 \pi i b Y_{j}\right) \mathrm{d} Y_{j} \\
J_{2}=\int_{-\infty}^{\infty}\left|g_{j}\right|^{2} h\left(Z_{j}\right) \mathrm{d} Z_{j} & (15 b) & F_{y}=\int_{-\infty}^{\infty} H\left(Y_{j}\right) \exp \left(-2 \pi i b Y_{j}\right) \mathrm{d} Y_{j}
\end{array}
$$




$$
\begin{aligned}
& \gamma_{y}=\int_{-\infty}^{\infty} f_{j} H\left(Y_{j}\right) \mathrm{d} Y_{j} \\
& A_{z}=\int_{-\infty}^{\infty} g_{j}^{*} h\left(Z_{j}\right) \exp \left(-2 \pi i b Z_{j}\right) \mathrm{d} Z_{j} \\
& F_{z}=\int_{-\infty}^{\infty} h\left(Z_{j}\right) \exp \left(-2 \pi i b Z_{j}\right) \mathrm{d} Z_{j} \\
& B_{z}=\int_{-\infty}^{\infty} g_{j} h\left(Z_{j}\right) \mathrm{d} Z_{j}
\end{aligned}
$$

Equations $15 \mathrm{a}-15 \mathrm{~h}$ indicate that since $f_{j}$ and $g_{j}$ are the functions of $Y_{j}$ and $Z_{j}$, respectively, the values taken by $Y_{j}$ and $Z_{j}$ are given statistically by the distribution functions $H\left(Y_{j}\right)$ and $h\left(Z_{j}\right)$, respectively. The intensity expression in eq 8 is rather complicated in comparison with that in the previous model. ${ }^{3}$ This is due to the introduction of orientational fluctuations of the lamellar assembly as well as the lamellar length $\bar{L}$ as shown in Figure 1. The terms $I_{1}^{\mathrm{B}}$ and $I_{1}^{\mathrm{C}}$ are similar to the terms $I_{\mathrm{B}}$ and $I_{\mathrm{C}}$ derived by Blundell. ${ }^{6}$

\section{The Trapezium Model}

In order to carry out numerical calculations, we now introduce a trapezium model (Figure 2(b)) to specify the density profile between two phases instead of an arbitrary function (Figure 2(a)). As reported by the previous paper, ${ }^{3}$ the density distribution $\zeta_{1}\left(y_{j}\right)$ of the $j$-th lamella (domain) with the thickness $Y_{j}$, denoting the positive density deviation from the average density of the system, is given by

$$
\begin{aligned}
\zeta_{1}\left(y_{j}\right)= & \frac{\left(\rho_{\mathrm{c}}-\bar{\rho}\right)}{t} y_{j}, \quad 0 \leqq y_{j} \leqq t \\
& \rho_{\mathrm{c}}-\bar{\rho}, \quad t<y_{j} \leqq\left(Y_{j}-t\right) \\
& \left(\rho_{\mathrm{c}}-\bar{\rho}\right)\left(Y_{j}-y_{j}\right) / t, \quad\left(Y_{j}-t\right)<y_{j} \leqq Y_{j}
\end{aligned}
$$

where $\rho_{\mathrm{c}}$ is the electron density of the lamella (domain) with the thickness $Y_{j}$ and $\bar{\rho}$ is the average electron density of the whole sample. Similarly, the density distribution $\zeta_{2}\left(z_{j}\right)$ of the medium with the length $Z_{j}$ surrounded by the $j$ - and $(j+1)$-th lamellae denotes the negative deviation, which is given by

$$
\begin{aligned}
\zeta_{2}\left(z_{j}\right)= & \frac{\left(\rho_{\mathrm{a}}-\bar{\rho}\right)}{t^{\prime}} z_{j}, \quad 0 \leqq z_{j} \leqq t^{\prime} \\
& \rho_{\mathrm{a}}-\bar{\rho}, \quad t^{\prime}<z_{j} \leqq\left(Z_{j}-t^{\prime}\right) \\
& \frac{\left(\rho_{\mathrm{a}}-\bar{\rho}\right)\left(Z_{j}-z_{j}\right)}{t^{\prime}}, \quad\left(Z_{j}-t^{\prime}\right)<z_{j} \leqq Z_{j}
\end{aligned}
$$

where $\rho_{\mathrm{a}}$ is the electron density of the lamella (domain) with the length $Z_{j}$.

According to the geometrical arrangement shown in Figure 3(b), we can obtain the following relations:

$$
\begin{aligned}
& \left(\rho_{\mathrm{c}}-\bar{\rho}\right)(\bar{Y}-t)=\left(\bar{\rho}-\rho_{\mathrm{a}}\right)\left(\bar{Z}-t^{\prime}\right) \\
& t^{\prime} / t=\left(\bar{\rho}-\rho_{\mathrm{a}}\right) /\left(\rho_{\mathrm{c}}-\bar{\rho}\right)
\end{aligned}
$$

The average electron density $\bar{\rho}$ corresponding to the average density of specimen can be written by using volume crystallinity $X_{\mathrm{c}}$ (or volume fraction) as follows:

$$
\bar{\rho}=X_{\mathrm{c}} \rho_{\mathrm{c}}+\left(1-X_{\mathrm{c}}\right) \rho_{\mathrm{a}}
$$

Thus, $t$ and $t^{\prime}$ can be obtained as a function of $X_{\mathrm{c}}$.

$$
t=\frac{\left(1-X_{\mathrm{c}}\right)\left(p-X_{\mathrm{c}}\right)}{1-2 X_{\mathrm{c}}} \bar{X}
$$

and

$$
t^{\prime}=\frac{X_{\mathrm{c}}\left(p-X_{\mathrm{c}}\right)}{1-2 X_{\mathrm{c}}} \bar{X}
$$

$p$ is given by $\bar{Y} / \bar{X}$.

Following Blundell, ${ }^{6}$ we assume that the variations of the lengths $Y_{j}$ and $Z_{j}$ are independent and are given by symmetrical Gaussian functions with the respective mean thicknesses of $\bar{Y}$ and $\bar{Z}$, and the standard deviations $\sigma_{y}$ and $\sigma_{z}$; thus

$$
H\left(Y_{j}\right)=(2 \pi)^{-1 / 2} \sigma_{\mathrm{y}}^{-1} \exp \left[-\left(Y_{j}-\bar{Y}\right)^{2} / 2 \sigma_{y}^{2}\right]
$$

$$
h\left(Z_{j}\right)=(2 \pi)^{-1 / 2} \sigma_{z}^{-1} \exp \left[-\left(Z_{j}-\bar{Z}\right)^{2} / 2 \sigma_{z}^{2}\right]
$$

By using $\zeta_{1}\left(y_{j}\right), \zeta_{2}\left(z_{j}\right), H\left(Y_{j}\right), h\left(Z_{j}\right), \bar{\rho}, t$, and $t^{\prime}, I_{i}^{\mathrm{B}}(i=1 \sim 3)$ and $I_{i}^{\mathrm{C}}(i=1 \sim 3)$ can be written as follows: 


$$
\begin{aligned}
& I_{1}^{\mathrm{B}}=\int_{0}^{2 \pi} p(\alpha)\left[\frac{1}{2 \bar{X} \bar{L}}\left\{\frac{\left(\rho_{\mathrm{c}}-\rho_{\mathrm{a}}\right)\left(1-2 X_{\mathrm{c}}\right)}{\pi^{2} a b\left(p-X_{\mathrm{c}}\right) \bar{X}} \sin (\pi a \bar{L}) \sin \left[\pi b\left(1-X_{\mathrm{c}}\right)\left(p-X_{\mathrm{c}}\right) \bar{X} /\left(1-2 X_{\mathrm{c}}\right)\right]\right\}^{2}\right. \\
& \times\left\{1-\left|F_{x}\right|^{2}+\left|F_{y}\right|\left(\left|F_{z}\right|^{2}-1\right) \cos \left[2 \pi b X_{c}\left(1-X_{c}-p\right) \bar{X} /\left(1-2 X_{c}\right)\right]\right. \\
& \left.+\left|F_{z}\right|\left(\left|F_{y}\right|^{2}-1\right) \cos \left[2 \pi b\left(1-3 X_{\mathrm{c}}+p X_{\mathrm{c}}+X_{\mathrm{c}}^{2}\right) \bar{X} /\left(1-2 X_{\mathrm{c}}\right)\right]\right\} / \\
& \left.\times\left[1+\left|F_{x}\right|^{2}-2\left|F_{x}\right| \cos (2 \pi b \bar{X})\right]\right] \mathrm{d} \alpha \\
& I_{1}^{\mathrm{C}}=\int_{0}^{2 \pi} p(\alpha)\left[\frac{2}{N \bar{X} \bar{L}}\left\{\frac{\left(\rho_{\mathrm{c}}-\rho_{\mathrm{a}}\right)\left(1-2 X_{\mathrm{c}}\right)}{2 \pi^{2} a b\left(p-X_{\mathrm{c}}\right) \bar{X}} \sin (a \pi \bar{L}) \sin \left[\pi b\left(1-X_{\mathrm{c}}\right)\left(p-X_{\mathrm{c}}\right) \bar{X} /\left(1-2 X_{\mathrm{c}}\right)\right]\right\}^{2}\right. \\
& \times\left[A_{1}-A_{1}\left|F_{x}\right|^{N} \cos (2 \pi N b \bar{X})-B_{1}\left|F_{x}\right|^{N} \sin (2 \pi N b \bar{X})\right] / \\
& \left.\times\left[1+\left|F_{x}\right|^{2}-2\left|F_{x}\right| \cos (2 \pi b \bar{X})\right]^{2}\right] \mathrm{d} \alpha \\
& I_{2}^{\mathrm{B}}=\int_{0}^{2 \pi} p(\alpha)\left[\frac{1}{2 \bar{X} \bar{L}}\left\{\frac{\left(\rho_{\mathrm{c}}-\rho_{\mathrm{a}}\right)\left(1-2 X_{\mathrm{c}}\right)}{\pi^{2} a b\left(p-X_{\mathrm{c}}\right) \bar{X}} \sin (\pi a \bar{L}) \sin \left[\pi b\left(1-X_{\mathrm{c}}\right)\left(p-X_{\mathrm{c}}\right) \bar{X} /\left(1-2 X_{\mathrm{c}}\right)\right]\right\}^{2}\right. \\
& \times\left\{1-\left|F_{x}\right|^{2}+\left|F_{z}\right|\left(\left|F_{y}\right|^{2}-1\right) \cos \left[2 \pi b\left(1-X_{c}\right)\left(1-X_{c}-p\right) \bar{X} /\left(1-2 X_{c}\right)\right]\right. \\
& \left.+\left|F_{y}\right|\left(\left|F_{z}\right|^{2}-1\right) \cos \left[2 \pi b\left(p-p X_{c}-X_{c}^{2}\right) \bar{X} /\left(1-2 X_{c}\right)\right]\right\} /\left[1+\left|F_{x}\right|^{2}\right. \\
& \left.\left.-2\left|F_{x}\right| \cos (2 \pi b \bar{X})\right]\right] \mathrm{d} \alpha \\
& I_{2}^{\mathrm{C}}=\int_{0}^{2 \pi} p(\alpha)\left[\frac{2}{N \bar{X} \bar{L}}\left\{\frac{\left(\rho_{\mathrm{c}}-\rho_{\mathrm{a}}\right)\left(1-2 X_{\mathrm{c}}\right)}{2 \pi^{2} a b\left(p-X_{\mathrm{c}}\right) \bar{X}} \sin (\pi a \bar{L}) \sin \left[\pi b X_{\mathrm{c}}\left(p-X_{\mathrm{c}}\right) /\left(1-2 X_{\mathrm{c}}\right)\right]\right\}^{2}\right. \\
& \times\left[A_{2}-A_{2}\left|F_{x}\right|^{N} \cos (2 \pi N b \bar{X})-B_{2}\left|F_{x}\right|^{N} \sin (2 \pi N b \bar{X})\right] /\left[1+\left|F_{x}\right|^{2}\right. \\
& \left.\left.-2\left|F_{x}\right| \cos (2 \pi b \bar{X})\right]^{2}\right] \mathrm{d} \alpha \\
& I_{3}^{\mathrm{B}}=\int_{0}^{2 \pi} p(\alpha)\left[\frac{1}{\bar{X} \bar{L}}\left\{\frac{\left(\rho_{\mathrm{c}}-\rho_{\mathrm{a}}\right)\left(1-2 X_{\mathrm{c}}\right)}{\pi^{2} a b\left(p-X_{\mathrm{c}}\right) \bar{X}}\right\}^{2} \sin (\pi a \bar{L}) \sin \left[\pi b\left(1-X_{\mathrm{c}}\right)\left(p-X_{\mathrm{c}}\right) \bar{X} /\left(1-2 X_{\mathrm{c}}\right)\right]\right. \\
& \times \sin \left[\pi b X_{\mathrm{c}}\left(p-X_{\mathrm{c}}\right) \bar{X} /\left(1-2 X_{\mathrm{c}}\right)\right]\left\{\left(1-\left|F_{x}\right|^{2}\right) \cos \left[\pi b\left(p-X_{\mathrm{c}}\right) \bar{X} /\left(1-2 X_{\mathrm{c}}\right)\right]\right. \\
& \left.+\left|F_{y}\right|\left(\left|F_{z}\right|^{2}-1\right) \cos \left[\pi b\left(X_{c}+p\right) \bar{X}\right]+\left|F_{z}\right|\left(\left|F_{y}\right|^{2}-1\right) \cos \left[\pi b\left(2-X_{c}-p\right) \bar{X}\right]\right\} / \\
& \left.\times\left[1+\left|F_{x}\right|^{2}-2\left|F_{x}\right| \cos (2 \pi b \bar{X})\right]\right] \mathrm{d} \alpha
\end{aligned}
$$

and

$$
\begin{aligned}
I_{3}^{\mathrm{C}}= & \int_{0}^{2 \pi} p(\alpha)\left[\frac{2}{N \bar{X} \bar{L}}\left\{\frac{\left(\rho_{\mathrm{c}}-\rho_{\mathrm{a}}\right)\left(1-2 X_{\mathrm{c}}\right)}{2 \pi^{2} a b\left(p-X_{\mathrm{c}}\right) \bar{X}}\right\}^{2} \sin (\pi a \bar{L}) \sin \left[\pi b\left(1-X_{\mathrm{c}}\right)\left(p-X_{\mathrm{c}}\right) \bar{X} /\left(1-2 X_{\mathrm{c}}\right)\right]\right. \\
& \times \sin \left[\pi b X_{\mathrm{c}}\left(p-X_{\mathrm{c}}\right) \bar{X} /\left(1-2 X_{\mathrm{c}}\right)\right]\left[A_{3}-A_{3}\left|F_{x}\right|^{N} \cos (2 \pi N b \bar{X})\right.
\end{aligned}
$$


$\left.\left.-B_{3}\left|F_{x}\right|^{N} \sin (2 \pi N b \bar{X})\right] /\left[1+\left|F_{x}\right|^{2}-2\left|F_{x}\right| \cos (2 \pi b \bar{X})\right]^{2}\right] \mathrm{d} \alpha$

where

$$
\begin{aligned}
& A_{1}=2\left|F_{x}\right|\left(\left|F_{x}\right|^{2}+1\right) \cos (2 \pi b \bar{X}) \\
& -\left|F_{x} \| F_{y}\right|\left(\left|F_{z}\right|^{2}+1\right) \cos \left[2 \pi b\left(X_{\mathrm{c}}^{2}+X_{\mathrm{c}}+X_{\mathrm{c}} p-1\right) \bar{X} /\left(1-2 X_{\mathrm{c}}\right)\right] \\
& -\left|F_{z}\right|\left(1+\left|F_{x}\right|^{2}\left|F_{y}\right|^{2}\right) \cos \left[2 \pi b\left(x_{\mathrm{c}}^{2}-3 X_{\mathrm{c}}+X_{\mathrm{c}} p+1\right) \bar{X} /\left(1-2 X_{\mathrm{c}}\right)\right] \\
& +2\left|F_{x} \| F_{z}\right|\left(1+\left|F_{y}\right|^{2}\right) \cos \left[2 \pi b X_{c}\left(1-X_{c}-p\right) \bar{X} /\left(1-2 X_{c}\right)\right]-4\left|F_{x}\right|^{2} \\
& B_{1}=\left|F_{x} \| F_{y}\right|\left(\left|F_{z}\right|^{2}-1\right) \sin \left[2 \pi b\left(X_{\mathrm{c}}^{2}+X_{\mathrm{c}}+X_{\mathrm{c}} p-1\right) \bar{X} /\left(1-2 X_{\mathrm{c}}\right)\right] \\
& -2\left|F_{x}\right|\left(1-\left|F_{x}\right|^{2}\right) \sin (2 \pi b \bar{X}) \\
& +\left|F_{z}\right|\left(1-\left|F_{x}\right|^{2}\left|F_{y}\right|^{2}\right) \sin \left[2 \pi b\left(X_{\mathrm{c}}^{2}-3 X_{\mathrm{c}}+X_{\mathrm{c}} p+1\right) \bar{X} /\left(1-2 X_{\mathrm{c}}\right)\right] \\
& -2\left|F_{x}\right|\left|F_{z}\right|\left(\left|F_{y}\right|^{2}-1\right) \sin \left[2 \pi b X_{c}\left(1-X_{\mathrm{c}}-p\right) \bar{X} /\left(1-2 X_{\mathrm{c}}\right)\right] \\
& A_{2}=2\left|F_{x}\right|\left(\left|F_{x}\right|^{2}+1\right) \cos (2 \pi b \bar{X}) \\
& -\left|F_{y}\right|\left(\left|F_{x}\right|^{2}\left|F_{z}\right|^{2}+1\right) \cos \left[2 \pi b\left(p-X_{\mathrm{c}} p-X_{\mathrm{c}}^{2}\right) \bar{X} /\left(1-2 X_{\mathrm{c}}\right)\right] \\
& -\left|F_{x} \| F_{z}\right|\left(\left|F_{y}\right|^{2}+1\right) \cos \left[2 \pi b\left(X_{\mathrm{c}}^{2}-4 X_{\mathrm{c}}+X_{\mathrm{c}} p-p+2\right) \bar{X} /\left(1-2 X_{\mathrm{c}}\right)\right] \\
& +2\left|F_{x}\right|\left|F_{y}\right|\left(1+\left|F_{z}\right|^{2}\right) \cos \left[2 \pi b\left(1-X_{c}\right)\left(1-X_{c}-p\right) \bar{X} /\left(1-2 X_{c}\right)\right]-4\left|F_{x}\right|^{2} \\
& B_{2}=2\left|F_{x}\right|\left(\left|F_{x}\right|^{2}-1\right) \sin (2 \pi b \bar{X}) \\
& +\left|F_{y}\right|\left(1-\left|F_{x}\right|^{2}\left|F_{z}\right|^{2}\right) \sin \left[2 \pi b\left(p-X_{\mathrm{c}} p-X_{\mathrm{c}}^{2}\right) \bar{X} /\left(1-2 X_{\mathrm{c}}\right)\right] \\
& +\left|F_{x}\right|\left|F_{z}\right|\left(1-\left|F_{y}\right|^{2}\right) \sin \left[2 \pi b\left(X_{\mathrm{c}}^{2}-4 X_{\mathrm{c}}+X_{\mathrm{c}} p-p+2\right) \bar{X} /\left(1-2 X_{\mathrm{c}}\right)\right] \\
& -2\left|F_{x}\right|\left|F_{y}\right|\left(\left|F_{z}\right|^{2}-1\right) \sin \left[2 \pi b\left(1-X_{c}\right)\left(1-X_{c}-p\right) \bar{X} /\left(1-2 X_{c}\right)\right] \\
& A_{3}=-\left|F_{x}\right|\left(1+\left|F_{x}\right|^{2}\right) \cos \left[\pi b\left(2-3 X_{\mathrm{c}}-p\right) \bar{X} /\left(1-2 X_{\mathrm{c}}\right)\right] \\
& +\left(\left|F_{x}\right|^{4}-4\left|F_{x}\right|^{2}+1\right) \cos \left[\pi b\left(X_{\mathrm{c}}-p\right) \bar{X} /\left(1-2 X_{\mathrm{c}}\right)\right] \\
& +\left(2\left|F_{x}\right|^{2}\left|F_{z}\right|-\left|F_{z}\right|+2\left|F_{x}\right|\left|F_{y}\right|-\left|F_{x}\right|^{3}\left|F_{y}\right|\right) \cos \left[\pi b\left(X_{c}+p-2\right) \bar{X}\right] \\
& +\left(2\left|F_{x}\right|^{2}\left|F_{y}\right|-\left|F_{y}\right|+2\left|F_{x}\right|\left|F_{z}\right|-\left|F_{x}\right|^{3}\left|F_{z}\right|\right) \cos \left[\pi b\left(X_{c}+p\right) \bar{X}\right] \\
& +2\left|F_{x}\right|^{2} \cos \left[\pi b\left(4-7 X_{\mathrm{c}}-p\right) \bar{X} /\left(1-2 X_{\mathrm{c}}\right)\right] \\
& +\left|F_{x}\right|\left(\left|F_{x}\right|^{2}+1\right) \cos \left[\pi b\left(5 X_{\mathrm{c}}-p-2\right) \bar{X} /\left(1-2 X_{\mathrm{c}}\right)\right] \\
& -\left|F_{x}\right|\left|F_{z}\right|\left(1+\left|F_{y}\right|^{2}\right) \cos \left[\pi b\left(X_{\mathrm{c}}+p-4\right) \bar{X}\right] \\
& -\left|F_{x}\right|\left|F_{y}\right|\left(1+\left|F_{z}\right|^{2}\right) \cos \left[\pi b\left(X_{\mathrm{c}}+p+2\right) \bar{X}\right] \\
& B_{3}=\left|F_{x}\right|\left(1-\left|F_{x}\right|^{2}\right) \sin \left[\pi b\left(5 X_{c}-p-2\right) \bar{X} /\left(1-2 X_{c}\right)\right] \\
& -3\left|F_{x}\right|\left(1-\left|F_{x}\right|^{2}\right) \sin \left[\pi b\left(2-3 X_{\mathrm{c}}-p\right) \bar{X} /\left(1-2 X_{\mathrm{c}}\right)\right] \\
& +\left(1-\left|F_{x}\right|^{4}\right) \sin \left[\pi b\left(X_{\mathrm{c}}-p\right) \bar{X} /\left(1-2 X_{\mathrm{c}}\right)\right] \\
& +\left|F_{x}\right|\left|F_{z}\right|\left(\left|F_{y}\right|^{2}-1\right) \sin \left[\pi b\left(X_{\mathrm{c}}+p-4\right) \bar{X}\right]
\end{aligned}
$$


where

$$
\begin{aligned}
& +\left[\left|F_{y}\right|\left(1-2\left|F_{x}\right|^{2}\right)-\left|F_{x}\right|\left|F_{z}\right|\left(\left|F_{x}\right|^{2}-2\right)\right] \sin \left[\pi b\left(X_{c}+p\right) \bar{X}\right] \\
& -\left[\left|F_{z}\right|\left(1-2\left|F_{x}\right|^{2}\right)-\left|F_{x}\right|\left|F_{y}\right|\left(\left|F_{x}\right|^{2}-2\right)\right] \sin \left[\pi b\left(X_{c}+p-2\right) \bar{X}\right] \\
& +\left|F_{x}\right|\left|F_{y}\right|\left(1-\left|F_{z}\right|^{2}\right) \sin \left[\pi b\left(X_{c}+p+2\right) \bar{X}\right]
\end{aligned}
$$

$$
\begin{aligned}
& \left|F_{y}\right|=\exp \left(-2 \pi^{2} b^{2} \sigma_{y}^{2}\right)=\exp \left[-2(\pi b \bar{Y})^{2}\left(\sigma_{y} / \bar{Y}\right)^{2}\right] \\
& \left|F_{z}\right|=\exp \left(-2 \pi^{2} b^{2} \sigma_{z}^{2}\right)=\exp \left[-2(\pi b \bar{Z})^{2}\left(\sigma_{z} / \bar{Z}\right)^{2}\right] \\
& \left|F_{x}\right|=\exp \left(-2 \pi^{2} b^{2} \sigma_{x}^{2}\right)=\exp \left[-2(\pi b \bar{X})^{2}\left(\sigma_{x} / \bar{X}\right)^{2}\right]
\end{aligned}
$$

and

$$
\sigma_{x}^{2}=\sigma_{y}^{2}+\sigma_{z}^{2}
$$

The result in eq 6-37 indicate that a twodimensional mathematical treatment by considering the distribution of lamellar thickness and orientation lamellar assemblies is essentially similar to the one-dimensional mathematical one discussed in the previous paper. ${ }^{3}$ However, in this paper, the effect of the two factors on the scattered intensity distribution can be discussed in terms of the azimuthal dependence of the intensity through similar numerical calculations. This estimation is un- derstood to be important to emphasize that the scattering must be due to positive and negative density deviations from the average density of the system.

We now wish to show that the expression for the scattered intensity derived for the trapezium model can be considerably simplified in the special case when $t=0$ and $t^{\prime}=0$. Then $p=X_{\mathrm{c}}$ and the following relations are obtained.

$$
\begin{aligned}
I_{\mathrm{B}}= & I_{1}^{\mathrm{B}}+I_{2}^{\mathrm{B}}+I_{3}^{\mathrm{B}} \\
= & \frac{1}{2 \bar{X} \bar{L}} \int_{0}^{2 \pi} p(\alpha)\left\{\frac{\left(\rho_{\mathrm{c}}-\rho_{\mathrm{a}}\right) \sin \pi a \bar{L}}{\pi^{2} a b}\right\}^{2}\left[\left\{1-\left|F_{x}\right|^{2}+\left|F_{y}\right|\left(\left|F_{z}\right|^{2}-1\right) \cos \left(2 \pi b X_{\mathrm{c}} \bar{X}\right)\right.\right. \\
& \left.\left.+\left|F_{z}\right|\left(\left|F_{y}\right|^{2}-1\right) \cos \left[2 \pi b\left(1-X_{\mathrm{c}}\right) \bar{X}\right]\right\} /\left[1+\left|F_{x}\right|^{2}-2\left|F_{x}\right| \cos (2 \pi b \bar{X})\right]\right] \mathrm{d} \alpha \\
I_{1}^{\mathrm{C}}= & \frac{1}{2 N \bar{X} \bar{L}} \int_{0}^{2 \pi} p(\alpha)\left\{\frac{\left(\rho_{\mathrm{c}}-\rho_{\mathrm{a}}\right)\left(1-X_{\mathrm{c}}\right) \sin \pi a \bar{L}}{\pi^{2} a b}\right\}^{2} \\
& \times\left[[ 1 - | F _ { x } | ^ { N } \operatorname { c o s } ( 2 \pi N b \overline { X } ) ] \left\{2\left|F_{x}\right|\left(\left|F_{x}\right|^{2}+1\right) \cos (2 \pi \mathrm{b} \bar{X})\right.\right. \\
& -\left|F_{x}\right|\left|F_{y}\right|\left(\left|F_{z}\right|^{2}+1\right) \cos \left[2 \pi b\left(1+X_{\mathrm{c}}\right) \bar{X}\right]+2\left|F_{x}\right|\left|F_{z}\right|\left(\left|F_{y}\right|^{2}+1\right) \cos \left(2 \pi b X_{\mathrm{c}} \bar{X}\right) \\
& \left.-\left|F_{z}\right|\left(1+\left|F_{x}\right|^{2}\left|F_{y}\right|^{2}\right) \cos \left[2 \pi b\left(1-X_{\mathrm{c}}\right) \bar{X}\right]-4\left|F_{x}\right|^{2}\right\} \\
& -\left|F_{x}\right|^{N} \sin (2 \pi N b \bar{X})\left\{2\left|F_{x}\right|\left(\left|F_{x}\right|^{2}-1\right) \sin (2 \pi b \bar{X})\right. \\
& -\left|F_{x}\right|\left|F_{y}\right|\left(\left|F_{z}\right|^{2}-1\right) \sin \left[2 \pi b\left(1+X_{\mathrm{c}}\right) \bar{X}\right]-2\left|F_{x}\right|\left|F_{z}\right|\left(\left|F_{y}\right|^{2}-1\right) \sin \left(2 \pi b X_{\mathrm{c}} \bar{X}\right) \\
& \left.\left.\left.-\left|F_{z}\right|\left(\left|F_{x}\right|^{2}\left|F_{y}\right|^{2}-1\right) \sin \left[2 \pi b\left(1-X_{\mathrm{c}}\right) \bar{X}\right]\right\}\right] /\left[1+\left|F_{x}\right|^{2}-2\left|F_{x}\right| \cos (2 \pi b \bar{X})\right]^{2}\right] \mathrm{d} \alpha
\end{aligned}
$$




$$
\begin{aligned}
& I_{2}^{\mathrm{C}}=\frac{1}{2 N \bar{X} \bar{L}} \int_{0}^{2 \pi} p(\alpha)\left\{\frac{\left(\rho_{\mathrm{c}}-\rho_{\mathrm{a}}\right) X_{\mathrm{c}} \sin \pi a \bar{L}}{\pi^{2} a b}\right\}^{2} \\
& \times\left[\left[[ 1 - | F _ { x } | ^ { N } \operatorname { c o s } ( 2 \pi N b \overline { X } ) ] \left\{2\left|F_{x}\right|\left(\left|F_{x}\right|^{2}+1\right) \cos (2 \pi b \bar{X})\right.\right.\right. \\
& -\left|F_{y}\right|\left(\left|F_{x}\right|^{2}\left|F_{z}\right|^{2}+1\right) \cos \left(2 \pi b X_{c} \bar{X}\right)+2\left|F_{x}\right|\left|F_{y}\right|\left(1+\left|F_{z}\right|^{2}\right) \cos \left[2 \pi b\left(1-X_{\mathrm{c}}\right) \bar{X}\right] \\
& \left.-\left|F_{x}\right|\left|F_{z}\right|\left(\left|F_{y}\right|^{2}+1\right) \cos \left[2 \pi b\left(2-X_{\mathrm{c}}\right) \bar{X}\right]-4\left|F_{x}\right|^{2}\right\} \\
& -\left|F_{x}\right|^{N} \sin (2 \pi N b \bar{X})\left\{2\left|F_{x}\right|\left(\left|F_{x}\right|^{2}-1\right) \sin (2 \pi b \bar{X})\right. \\
& +\left|F_{y}\right|\left(1-\left|F_{x}\right|^{2}\left|F_{z}\right|^{2}\right) \sin \left(2 \pi b X_{c} \bar{X}\right)-2\left|F_{x}\right|\left|F_{y}\right|\left(\left|F_{z}\right|^{2}-1\right) \sin \left[2 \pi b\left(1-X_{c}\right) \bar{X}\right] \\
& \left.\left.\left.-\left|F_{x}\right|\left|F_{z}\right|\left(\left|F_{y}\right|^{2}-1\right) \sin \left[2 \pi b\left(2-X_{c}\right) \bar{X}\right]\right\}\right] /\left[1+\left|F_{x}\right|^{2}-2\left|F_{x}\right| \cos (2 \pi b \bar{X})\right]^{2}\right] \mathrm{d} \alpha \\
& I_{3}^{\mathrm{C}}=\frac{1}{4 N \bar{X} \bar{L}} \int_{0}^{2 \pi} p(\alpha)\left\{\frac{\left(\rho_{\mathrm{c}}-\rho_{\mathrm{a}}\right) \sin \pi a \bar{L}}{\pi^{2} a b}\right\}^{2} \\
& \times X_{\mathrm{c}}\left(1-X_{\mathrm{c}}\right)\left[\left[[ 1 - | F _ { x } | ^ { N } \operatorname { c o s } ( 2 \pi N b \overline { X } ) ] \left\{2\left|F_{x}\right|^{2} \cos (4 \pi b \bar{X})\right.\right.\right. \\
& -\left|F_{x}\right|\left|F_{y}\right|\left(1+\left|F_{z}\right|^{2}\right) \cos \left[2 \pi b\left(1+X_{c}\right) \bar{X}\right] \\
& +\left(2\left|F_{x}\right|^{2}\left|F_{y}\right|-\left|F_{y}\right|+2\left|F_{x}\right|\left|F_{z}\right|-\left|F_{x}\right|^{3}\left|F_{z}\right|\right) \cos \left(2 \pi b X_{\mathrm{c}} \bar{X}\right) \\
& +\left(2\left|F_{x}\right|^{2}\left|F_{z}\right|-\left|F_{z}\right|+2\left|F_{x}\right|\left|F_{y}\right|-\left|F_{x}\right|^{3}\left|F_{y}\right|\right) \cos \left[2 \pi b\left(1-X_{c}\right) \bar{X}\right] \\
& \left.-\left|F_{x}\right|\left|F_{z}\right|\left(\left|F_{y}\right|^{2}+1\right) \cos \left[2 \pi b\left(2-X_{c}\right) \bar{X}\right]+\left|F_{x}\right|^{4}-4\left|F_{x}\right|^{2}+1\right\} \\
& -\left|F_{x}\right|^{N} \sin (2 \pi N b \bar{X})\left\{4\left|F_{x}\right|\left(\left|F_{x}\right|^{2}-1\right) \sin (2 \pi b \bar{X})\right. \\
& -\left|F_{x}\right|\left|F_{z}\right|\left(\left|F_{y}\right|^{2}-1\right) \sin \left[2 \pi b\left(2-X_{c}\right) \bar{X}\right] \\
& +\left|F_{x}\right|\left|F_{y}\right|\left(1-\left|F_{z}\right|^{2}\right) \sin \left[2 \pi b\left(1+X_{\mathrm{c}}\right) \bar{X}\right] \\
& +\left(\left|F_{y}\right|-2\left|F_{x}\right|^{2}\left|F_{y}\right|+2\left|F_{x}\right|\left|F_{z}\right|-\left|F_{x}\right|^{3}\left|F_{z}\right|\right) \sin \left(2 \pi b X_{\mathrm{c}} \bar{X}\right) \\
& \left.\left.+\left(2\left|F_{x}\right|\left|F_{y}\right|-\left|F_{x}\right|^{3}\left|F_{y}\right|+\left|F_{z}\right|-2\left|F_{x}\right|^{2}\left|F_{z}\right|\right) \sin \left[2 \pi b\left(1-X_{c}\right) \bar{X}\right]\right\}\right] / \\
& \left.\times\left[1+\left|F_{x}\right|^{2}-2\left|F_{x}\right| \cos (2 \pi b \bar{X})\right]^{2}\right] \mathrm{d} \alpha
\end{aligned}
$$

Equation 38 is of a similar form which was derived by Blundell ${ }^{6}$ and $I_{1}^{\mathrm{C}}$ in eq 39 is also similar to $I_{\mathrm{C}}$ which was obtained by Hashimoto et al. ${ }^{11}$ The terms $I_{2}^{\mathrm{C}}$ and $I_{3}^{\mathrm{C}}$ are very important for understanding the SAXS intensity distribution for real systems, as will be discussed later.

We now consider the simple case where the thicknesses $Y_{j}$ and $Z_{j}(j=1 \sim N)$ have constant values $\bar{Y}$ and $\bar{Z}$, respectively. In this case, the standard deviations $\sigma_{y}$ and $\sigma_{z}$ are zero and therefore we obtain $\left|F_{x}\right|=\left|f_{y}\right|=\left|F_{z}\right|=1$. It follows that

$$
I_{\mathrm{B}}=I_{1}^{\mathrm{B}}=I_{2}^{\mathrm{B}}=I_{3}^{\mathrm{B}}=0 \quad \text { at } s \bar{X} \neq n(n, \text { integer })
$$

However, if $s \bar{X}=n\left(n\right.$, integer) and $\sigma_{y}=\sigma_{z}=0$, we cannot define the term $I_{\mathrm{B}}$ mathematically. Therefore, it should be understood that $I /(N \bar{L} \bar{X})$ can be represented solely by the term, $-I_{\mathrm{C}}=-\left(I_{1}^{\mathrm{C}}+I_{2}^{\mathrm{C}}+I_{3}^{\mathrm{C}}\right)$, and we have 


$$
\begin{aligned}
I / N \bar{L} \bar{X}= & -I_{\mathrm{C}}=-\left(I_{1}^{\mathrm{C}}+I_{2}^{\mathrm{C}}+I_{3}^{\mathrm{C}}\right) \\
= & \int_{0}^{2 \pi} p(\alpha)\left\{\frac{\left(\rho_{\mathrm{c}}-\rho_{\mathrm{a}}\right)\left(1-2 X_{\mathrm{c}}\right) \sin \pi a \bar{L}}{(\pi b)^{2}\left(p-X_{\mathrm{c}}\right) \bar{X} \pi a}\right\}^{2} \frac{\sin ^{2}(\pi N b \bar{X})}{N \bar{X} \sin ^{2}(\pi b \bar{X})} \\
& \times\left\{\sin ^{2}\left[\pi b\left(1-X_{\mathrm{c}}\right)\left(p-X_{\mathrm{c}}\right) \bar{X} /\left(1-2 X_{\mathrm{c}}\right)\right] \sin ^{2}\left[\pi b X_{\mathrm{c}}\left(1-X_{\mathrm{c}}-p\right) \bar{X} /\left(1-2 X_{\mathrm{c}}\right)\right]\right. \\
& +\sin ^{2}\left[\pi b X_{\mathrm{c}}\left(p-X_{\mathrm{c}}\right) \bar{X} /\left(1-2 X_{\mathrm{c}}\right)\right] \sin ^{2}\left[\pi b\left(1-X_{\mathrm{c}}\right)\left(1-X_{\mathrm{c}}-p\right) \bar{X} /\left(1-2 X_{\mathrm{c}}\right)\right] \\
& -2 \sin \left[\pi b\left(1-X_{\mathrm{c}}\right)\left(p-X_{\mathrm{c}}\right) \bar{X} /\left(1-2 X_{\mathrm{c}}\right)\right] \sin \left[\pi b X_{\mathrm{c}}\left(p-X_{\mathrm{c}}\right) \bar{X} /\left(1-2 X_{\mathrm{c}}\right)\right] \\
& \times \sin \left[\pi b X_{\mathrm{c}}\left(1-X_{\mathrm{c}}-p\right) \bar{X} /\left(1-2 X_{\mathrm{c}}\right)\right] \sin \left[\pi b\left(1-X_{\mathrm{c}}\right)\left(1-X_{\mathrm{c}}-p\right) \bar{X} /\left(1-2 X_{\mathrm{c}}\right)\right] \\
& \times \cos (\pi b \bar{X})\} \mathrm{d} \alpha
\end{aligned}
$$

In Eq. (43), if $p=X_{\mathrm{c}}$, then is $t=0$ and $t^{\prime}=0$, we have

$$
\begin{aligned}
I / N \bar{L} \bar{X}= & -I_{\mathrm{C}} \\
= & \int_{0}^{2 \pi} p(\alpha)\left[\frac{\left(\rho_{\mathrm{c}}-\rho_{\mathrm{a}}\right)^{2} \sin ^{2}(\pi N b \bar{X}) \sin ^{2}(\pi a \bar{L}) \bar{X} \bar{L}}{N(\pi b \bar{X})^{2}(\pi a \bar{L})^{2} \sin ^{2}(\pi b \bar{X})}\right. \\
& \times\left\{\left(1-X_{\mathrm{c}}\right)^{2} \sin ^{2}\left(\pi b X_{\mathrm{c}} \bar{X}\right)+X_{\mathrm{c}}^{2} \sin ^{2}\left[\pi b\left(1-X_{\mathrm{c}}\right) \bar{X}\right]\right. \\
& \left.\left.-2\left(1-X_{\mathrm{c}}\right) X_{\mathrm{c}} \sin \left(\pi b X_{\mathrm{c}} \bar{X}\right) \sin \left[\pi b\left(1-X_{\mathrm{c}}\right) \bar{X}\right] \cos (\pi b \bar{X})\right\}\right] \mathrm{d} \alpha
\end{aligned}
$$

If the $I_{\mathrm{C}}$ term is considered in B-type models, the equation corresponding to eq 44 is given by

$$
\begin{aligned}
I / N \bar{X} \bar{L} & =-I_{\mathrm{C}} \\
& =\int_{0}^{2 \pi} p(\alpha) \frac{\left(\rho_{\mathrm{c}}-\rho_{\mathrm{a}}\right)^{2} \sin ^{2}(\pi N b \bar{X}) \sin ^{2}(\pi a \bar{L}) \bar{X} \bar{L}}{N(\pi b \bar{X})^{2}(\pi a \bar{L})^{2} \sin ^{2}(\pi b \bar{X})} \sin ^{2}\left(\pi b X_{\mathrm{c}} \bar{X}\right) \mathrm{d} \alpha
\end{aligned}
$$

Equation 45 is similar to the first term in eq 44.

Considering a real system, the portion of the specimen sampled by the $\mathrm{X}$-ray beam contains a distribution of the number $N$ of pairs of the two phases. This concept must be introduced in order to smear out the many subsidiary maxima which appear at lower scattering angle $s \bar{X}$. This has been taken into consideration by Hashimoto et al. ${ }^{11}$ by introducing a discrete symmetrical distribution of $N$ with standard deviation $\sigma_{N}=3$. This distribution is written as

$$
\begin{aligned}
P(N)= & \exp \left\{-\frac{(N-\bar{N})^{2}}{2 \sigma_{N}^{2}}\right\} / \sum_{N=1}^{2 \bar{N}-1} \\
& \times \exp \left\{-\frac{(N-\bar{N})^{2}}{2 \sigma_{N}^{2}}\right\}
\end{aligned}
$$

In the present work we assume the same type of distribution of $N$. Then the average value of the term $I_{\mathrm{C}}$ is given by

$$
\left\langle I_{\mathrm{C}}\right\rangle_{\mathrm{av}}=\sum_{N=1}^{2 \bar{N}-1} I_{\mathrm{C}} P(N)
$$

In our subsequent calculations, an average value of $N, \bar{N}$ is assumed to be 10 .

To complete this theoretical analysis we now discuss the function $p(\alpha) . p(\alpha)$ is a distribution function describing the orientation of the lamellae with respect to the $X_{3}$ axis. It is defined by

$$
p(\alpha)=\exp \left[-\sigma_{\alpha}^{2} \sin ^{2} \alpha\right]
$$

where $\sigma_{\alpha}$ is a parameter associated with the shape of $p(\alpha)$. When $\sigma_{\alpha}$ is large value, $p(\alpha)$ 
shows a sharp distribution. By contrast, when $\sigma_{\alpha}=0, p(\alpha)$ shows random distribution with maximum fluctuation.

The expressions for the SAXS intensity distribution, as formulated in eq $8-37$, contain five parameters $\sigma_{y} / \bar{Y}, \sigma_{z} / \bar{Z}, \bar{L}, p$, and $X_{\mathrm{c}}$ which must be determined by numerical calculation. The value of $\sigma_{x} / \bar{X}$, which is an important parameter to describe the fluctuation of the long period, can be obtained from eq 37 if $\sigma_{y} / \bar{Y}, \sigma_{z} / \bar{Z}$ and $p$ can be determined. In the case of a block (or graft) copolymer with alternating lamellar phases, $p$ and $X_{\mathrm{c}}$ (volume fraction) can respectively be determined in principle by observation under the electron microscope and from the monomer contents before polymerization. In the case of semicrystalline polymers, $X_{c}$ (the volume crystallinity) can be readily estimated, for example, by a density measurement but $p$ cannot be easily determined. However, in a real system, $t$ and $t^{\prime}$ are usually much smaller than $\bar{Y}$ and accordingly for numerical calculations, $p$ can be assumed essentially to be close to $X_{\mathrm{c}}$.

Consider first the difference of $I_{\mathrm{B}}$ and $I$ calculated from A- and B-type models, in the case when the parameters describing the fluctuations in the thickness of the crystalline and amorphous regions are set at very small values such as $\sigma_{y} / \bar{Y}=\sigma_{z} / \bar{Z}=0.001$. The $s \bar{X}$ dependence of $I_{\mathrm{B}}$ and $I$ obtained in the case of $\mu=$ $0^{\circ}$. For simplicity, the lamellae are assumed to be oriented perpendicular to the direction of the $X_{3}$ axis, that is, $\alpha=0^{\circ}$. Furthermore, the lengths $t$ and $t^{\prime}$ of the transition region are fixed at zero. This indicates a step function for the density variation between the crystalline and amorphous phases similar to that proposed by Wenig and Brämer. ${ }^{1}$ The crystallinity $X_{\mathrm{c}}(=\bar{Y} / \bar{X})$ is fixed to be 0.6 and $\bar{Y} / \bar{L}$ is also fixed as 0.02 .

Figure 4 shows the results. The curves on the left hand (Figure 4(a)) correspond to the model shown in Figure 2(b) (A-type), while the curves on the right hand (Figure 4(b)) correspond to the model shown in Figure 3(b) (B-

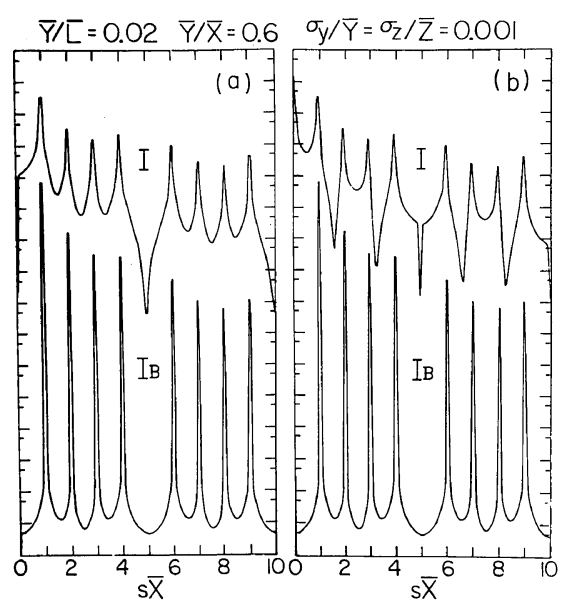

Figure 4. The profile of $I$ and $I_{\mathrm{B}}$ at $\sigma_{y} / \bar{Y}=\sigma_{z} / \bar{Z}=0.001$. (a) A-type; (b) B-type.

type). As demonstrated in eq 38 , the profile of $I_{\mathrm{B}}$ for A-type and that of B-type exhibit exactly the same shape. They have many maxima, but the maxima at $s \bar{X}=5$ and 10 are missing in both models. This arises from the characteristic property of the $I_{\mathrm{B}}$ term, which will be discussed below. By using the relations $\left|F_{x}\right|=\left|F_{y}\right|\left|F_{z}\right|, \quad\left|F_{y}\right|=\left|F_{z}\right|, \quad a=0$, and $b=s$, eq 38 can be rewritten as follows:

$$
\begin{aligned}
I_{\mathrm{B}}= & \frac{\bar{L} \bar{X}}{2}\left\{\frac{\rho_{\mathrm{c}}-\rho_{\mathrm{a}}}{\pi s \bar{X}}\right\}^{2} \\
& \times\left[\left\{1-\left|F_{y}\right|^{4}-2\left|F_{y}\right|\left(1-\left|F_{y}\right|^{2}\right)\right.\right. \\
& \left.\times \cos (\pi s \bar{X}) \cos \left[\pi s\left(2 X_{\mathrm{c}}-1\right) \bar{X}\right]\right\} / \\
& \left.\times\left\{1+\left|F_{y}\right|^{4}-2\left|F_{y}\right|^{2} \cos (2 \pi s \bar{X})\right\}\right]
\end{aligned}
$$

Equation 49 should have very large values when $s \bar{X}$ is integer and very small values when $s \bar{X}$ is $n / 2$ ( $n$, integer), taking into account the denominator of eq 49. When $X_{\mathrm{c}}=0.6$, $\cos (\pi s \bar{X}) \cos \left[\pi s\left(2 X_{c}-1\right) \bar{X}\right]$ has its maximum value (unity) at $s \bar{X}=5(2 n-1)$ (n, integer). Then eq 49 is reduced to

$$
I_{\mathrm{B}}=\frac{\bar{L} \bar{X}}{2}\left\{\frac{\rho_{\mathrm{c}}-\rho_{\mathrm{a}}}{\pi s \bar{X}}\right\}^{2}\left[\frac{1-\left|F_{y}\right|}{1+\left|F_{y}\right|}\right]
$$

This equation indicates that the $I_{\mathrm{B}}$ term has a very small value at $s \bar{X}=5$ and 10 as shown in 


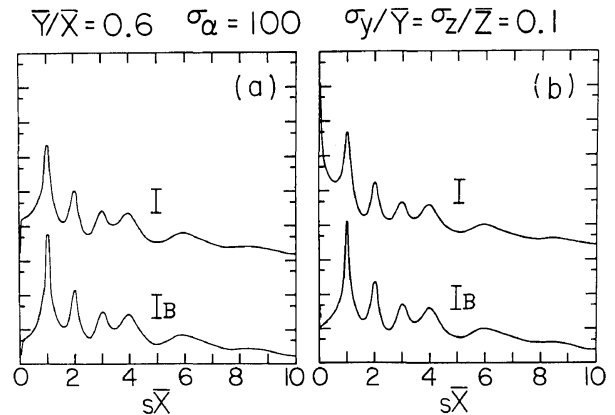

Figure 5. The profile of $I$ and $I_{\mathrm{B}}$ at $\sigma_{y} / \bar{Y}=\sigma_{z} / \bar{Z}=0.1$ and $\sigma_{\alpha}=100$. (a) A-type; (b) B-type.

Figure 4, because $\left|F_{y}\right|$ is close to unity for the given parameter $\sigma_{y} / \bar{Y}=0.001$. The peaks of $I$ at $s \bar{X}=n(n$, integer) are broader than those of $I_{\mathrm{B}}$, which is due to the characteristic property of the $I_{\mathrm{C}}$ term. The $I_{\mathrm{C}}$ term is presumably a smooth curve showing broader maxima at $s \bar{X}=n$ ( $n$, integer). A comparison of both the scattered intensity curves $I$ in Figures 4(a) and (b) in detail shows that the peaks as well as background for A-type are broader than those for B-type. This indicates that the $I_{2}^{\mathrm{C}}$ and $I_{3}^{\mathrm{C}}$ terms are reflected in the profile of $I$ for Atype, since the $I_{1}^{\mathrm{C}}$ term is equivalent to the $I_{\mathrm{C}}$ term derived from B-type at $t=t^{\prime}=0$. Incidentally, the effect of the lamellar length $\bar{L}$ causes no significant change in the $s \bar{X}$ dependence of the scattered intensity distribution at $\mu=0^{\circ}$, since $\left|F_{y}\right|$ is independent of $\bar{L}$ in eq 49 .

Figure 5 shows the profiles of $I$ and $I_{\mathrm{B}}$ when $\sigma_{y} / \bar{Y}=\sigma_{z} / \bar{Z}=0.1$ corresponding to a large fluctuation of the thickness of the crystalline and amorphous regions. The parameter $\sigma_{\alpha}$ is fixed to be 100 indicating that the lamellar assemblies are oriented almost perpendicular to the $X_{3}$ axis. The height of the maxima of $I$ and $I_{\mathrm{B}}$ decreases considerably at large values of $s \bar{X}$ and then both would be expected to become a smooth monotonically decreasing line with increase of $s \bar{X}$. This indicates that the increase of $\sigma_{y} / \bar{Y}$ and $\sigma_{z} / \bar{Z}$ makes the peak and background of $I$ and $I_{\mathrm{B}}$ broad. Comparing the profile of $I$ in Figures 5(a) and (b), both the shapes resemble each other except

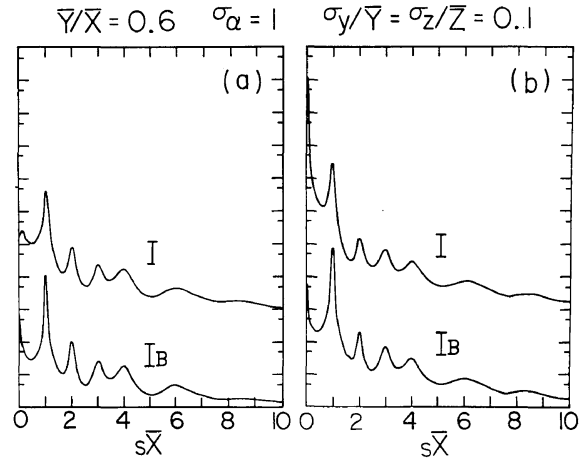

Figure 6. The profile of $I$ and $I_{\mathrm{B}}$ at $\sigma_{y} / \bar{Y}=\sigma_{z} / \bar{Z}=0.1$ and $\sigma_{\alpha}=1$. (a) A-type; (b) B-type.

on $0 \leqq s \bar{X} \leqq 1$. This is probably due to the $I_{\mathrm{C} 2}$ and $I_{\mathrm{C} 3}$ terms at $\sigma_{y} / \bar{Y}=\sigma_{z} / \bar{Z}=0.1$ which cause no significant change in the profile of $I$.

In order to consider the effect of orientation distribution of lamellar assemblies on the $s \bar{X}$ dependence of the scattered intensity distribution at $\mu=0^{\circ}$, the calculation was carried out at $\sigma_{\alpha}=1$ corresponding to the broad distribution. The result is shown in Figures 6(a) and (b). Both the shapes of $I$ resemble each other except on $0 \leqq s \bar{X} \leqq 1$. This tendency is similar to those which appeared in Figures 4 and 5. Therefore, the question can be arisen why the considerable difference of SAXS intensity distribution between A-type model in Figure 2(b) and B-type model in Figure 3(b) comes out in the range of $0 \leqq s \bar{X} \leqq 1$. As shown in Figures 4(a) and 5(a), both the intensities $I$ on A-type model decrease gradually as $s \bar{X}$ decreases in the range of $0 \leqq s \bar{X} \leqq 1$ and becomes zero at $s \bar{X}=0$, when the lamellar assemblies orient almost perpendicular to the $X_{3}$ axis. Furthermore, as shown in Figure 6(a), the intensity $I$ shows a small peak on $0 \leqq s \bar{X} \leqq 1$ and becomes zero at $s \bar{X}=0$, when the orientation of the lamellar assemblies is characteristic of a broad distribution. By contrast, the scattered intensities $I$ as shown in Figures 4(b), $5(\mathrm{~b})$, and 6(b), corresponding to B-type, increase as $s \bar{X}$ decreases in the range of $0 \leqq s \bar{X} \leqq 1$ and gives the zero-order scattering. The magnitude of $I$ at $s \bar{X}=0$ is proportional to $\bar{N}$. 
Accordingly, when $\bar{N}$ is infinite, the value of $I$ becomes infinite and therefore corresponds to the intensity of an incident beam automatically. However, the scattered intensity must be zero at $s \bar{X}=0$ in an ideal case. As for the experiment to observe the intensity distribution of SAXS, the distribution in usually estimated by substituting an unscattered intensity associated with an incident beam as well as an air scattering one from the observed intensity. However, such beams around $s \bar{X}=0$ are eliminated by a lead stopper and therefore nobody can demonstrate that the scattered intensity is zero at $s \bar{X}=0$, experimentally. In the very small angle region except around $s \bar{X}=0$, the observed intensity distribution at $\mu=0^{\circ}$ showed a gradual increasing curve with decreasing $s \bar{X},{ }^{12}$ when the measurement was carried out by using a PSPC (position sensitive proportional counter) system for undrawn dry gel film which was produced by gelation crystallization from dilute solutions according to the method of Smith and Lemstra ${ }^{13}$ and has drawability up to an elongation ratio of 20 times. ${ }^{8.9}$ A similar phenomenon has been observed for a sedimented mat of single crystals of polyethylene. Namely, the experimental result is similar to the calculated one shown in Figure 6(a) but different from the ones in Figure 4(a) or 5(a). This indicates that the orientation of assemblies constructed of alternating lamellae is not sharp but broad with respect to the $X_{3}$ axis (the film normal direction). Accordingly, the scattered intensity distribution calculated on A-type model in Figure 2(b) is better than that calculated on Btype one in Figure 3(b) in order to explain the scattering from real system. Furthermore, the validity of A-type can be demonstrated in terms of the concept that the equation of SAXS intensity satisfies Babinet's reciprocal theorem for volume fraction when there is no fluctuation in the thickness of the alternating lamellar phases. This concept is described in Appendix.

In order to understand the effect of the

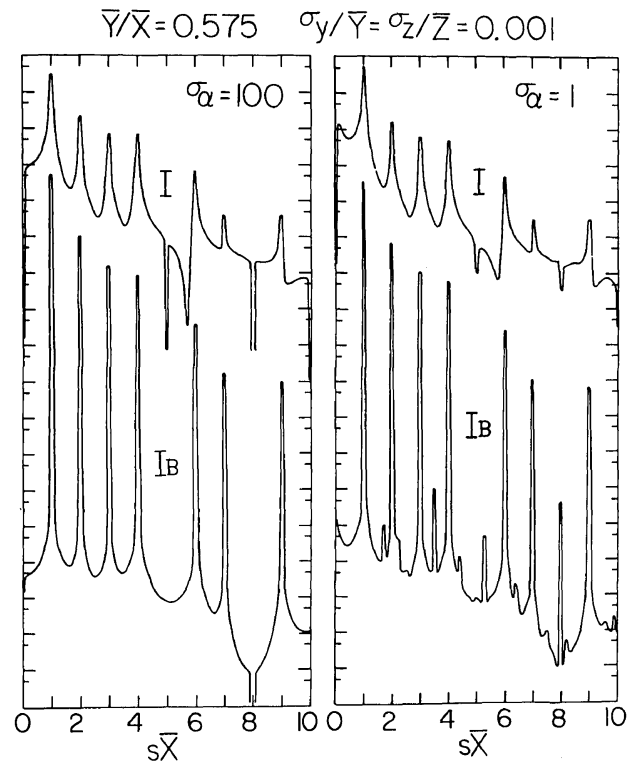

Figure 7. The effect of the orientation distribution function of lamellar assemblies, $\sigma_{\alpha}$, on $I$ and $I_{\mathrm{B}}$ calculated on A-type of $\sigma_{y} / \bar{Y}=\sigma_{z} / \bar{Z}=0.001$ and $t / \bar{X}=0.05$.

transition zones $t$ and $t^{\prime}$ on the scattered intensity, numerical calculation was carried out by using eq $24-29$. Figure 7 shows the effect of the transition zones on $I$ and $I_{\mathrm{B}}$, when $\sigma_{y} / \bar{Y}=\sigma_{z} / \bar{Z}=0.001$ corresponding to a small fluctuation of the thickness of the crystalline and amorphous regions. The calculation was carried out by using A-type model at $X_{\mathrm{c}}=0.6$. The ratio of the transition zone $t$ to the average repeat distance $\bar{X}$ is fixed as $t / \bar{X}=0.05$ and from eq $21, p=(\bar{Y} / \bar{X})$ becomes 0.575 . In this case, the ratio of $t^{\prime} / \bar{X}$ ia also determined to be 0.075 automatically from eq 21 and 22 . The parameter $\sigma_{\alpha}$ is fixed as 100 and 1 to characterize two extremes of the distribution $p(\alpha)$, the one narrow and the other broad. As illustrated in Figure 7 , the curves of $I_{\mathrm{B}}$ shows that maxima up to the tenth are missing. The distribution of $I_{\mathrm{B}}$ at $\sigma_{\alpha}=1$ shows many small peaks, which are not shown for $I_{\mathrm{B}}$ at $\sigma_{\alpha}=100$. This cause is unresolved problem. Such small peaks, however, cause no significant change in the profile of $I$. Interestingly, the profile of $I$ at $\sigma_{\alpha}=1$ is smoother than that at $\sigma_{\alpha}=100$. This 


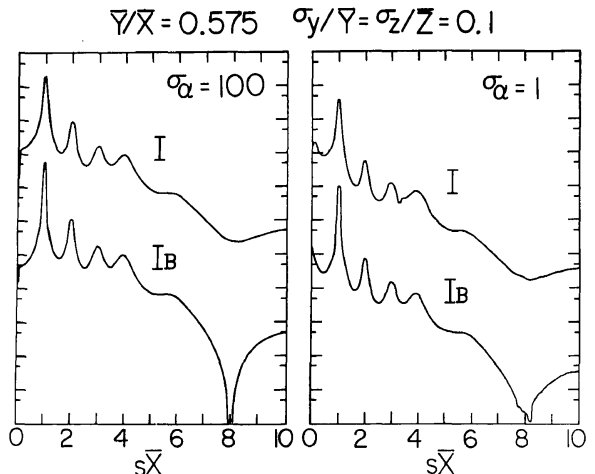

Figure 8. The effect of the orientation distribution function of lamellar assemblies, $\sigma_{\alpha}$, on $I$ and $I_{\mathrm{B}}$ calculated on A-type at $\sigma_{y} / \bar{Y}=\sigma_{z} / \bar{Z}=0.1$ and $t / \bar{X}=0.05$.

is presumably due to the addition to the $I_{\mathrm{C}}$ term which makes the peaks and background smooth at $\sigma_{\alpha}=1$.

Figure 8 shows the calculated results of $I$ and $I_{\mathrm{B}}$ at $t / \bar{X}=0.05$ when $\sigma_{y} / \bar{Y}=\sigma_{z} / \bar{Z}=0.1$ corresponding to a large fluctuation of the thickness of the crystalline and amorphous regions. The magnitude of $I_{\mathrm{B}}$ decreases considerably at $s \bar{X}=8$. This tendency of $I_{\mathrm{B}}$ is the same as the case in Figure 7 in spite of large value of $\sigma_{y} / \bar{Y}$ and $\sigma_{z} / \bar{Z}$. The profile of $I$ at $s \bar{X}=8$, however, shows a small trough around $s \bar{X}=8$. Furthermore, it should be noted that the profiles of $I_{\mathrm{B}}$ and $I$ show rapid decrease of the magnitude with increasing $s \bar{X}$ in comparison with the profiles shown in Figures 5(a) and 6(a). Such a rapid decrease was discussed already by Blundell ${ }^{6}$ and Hashimoto et al. ${ }^{11}$ Incidentally, the decrease is independent of the parameter $\sigma_{\alpha}$ associated with the orientation distribution of lamellar assemblies.

In order to demonstrate the validity of Atype, the azimuthal $\mu$-dependence of the scattered intensity was calculated. The results are shown in Figures 9-13. Among them, Figures 9-11 exhibit the patterns calculated from A-type and Figures 12 and 13 the patterns calculated from B-type. First, we shall discuss the $\mu$-dependence as the function of the parameters $\sigma_{y} / \bar{Y}, \sigma_{z} / \bar{Z}$, and $\sigma_{\alpha}$ in the case of $\bar{X} / \bar{L}=0.02$ corresponding to the system which

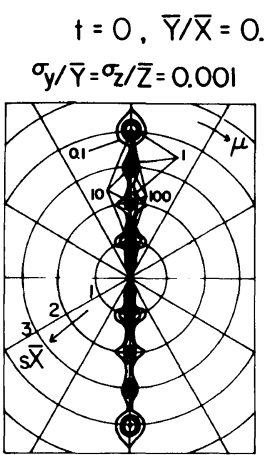

(a) $\sigma_{\alpha}=100$

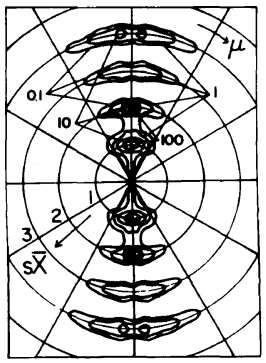

(b) $q=5$

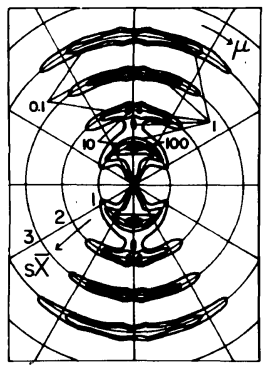

(c) $\sigma_{\alpha}=3$

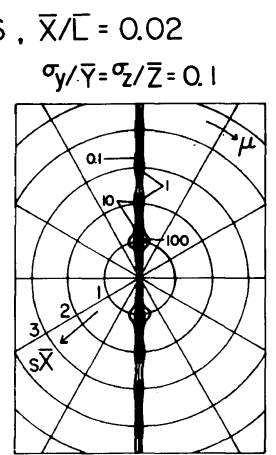

(d) $\sigma_{\alpha}=100$

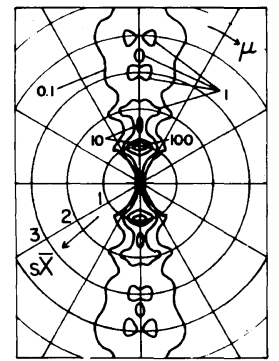

(e) $\sigma_{\alpha}=5$

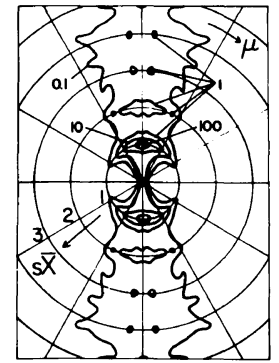

(f) $\sigma_{\alpha}=3$
Figure 9. SAXS patterns calculated on A-type at $t=0$ and $\bar{X} / \bar{L}=0.02$.

the lamellar length $\bar{L}$ is much longer than the average repeat distance $\bar{X}$. The result is shown in Figure 9. The profile of the scattered intensity for the $\mu$-dependence is affected by $\sigma_{\alpha}$. Judging from the change in profile with decreasing $\sigma_{\alpha}$, one would expect that the intensity distribution becomes broader as the distribution $p(\alpha)$ becomes broader. The increase of fluctuation in the thickness of the crystalline and amorphous regions, namely, the increase of $\sigma_{y} / \bar{Y}$ and $\sigma_{z} / \bar{Z}$ causes the disappearance of the third order peak intensity as far as the 


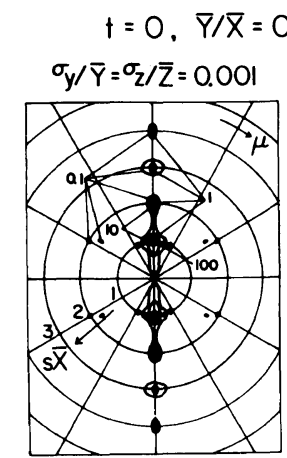

(a) $\sigma_{\alpha}=100$

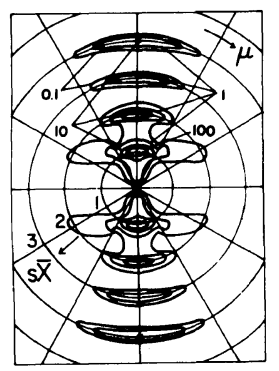

(b) $\sigma_{\alpha}=5$

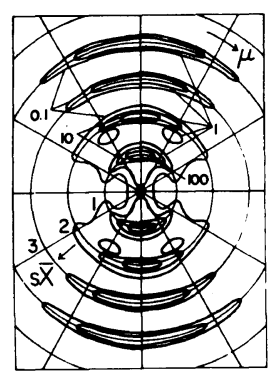

(c) $\sigma_{\alpha}=3$

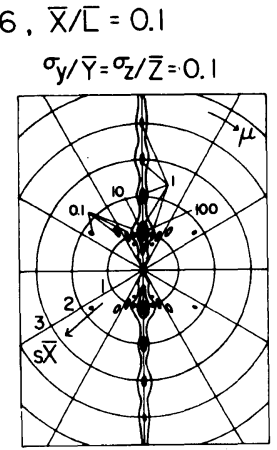

(d) $\sigma_{\alpha}=100$

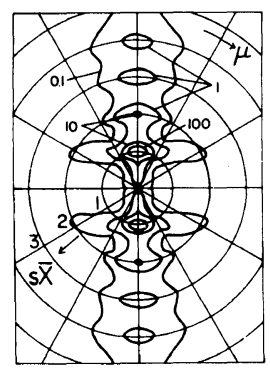

(e) $\sigma_{\alpha}=5$

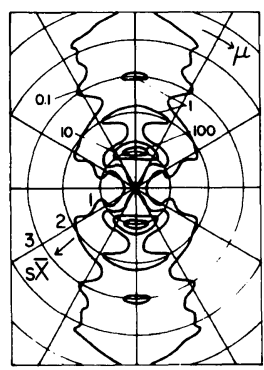

(f) $\sigma_{\alpha}=3$
Figure 10. SAXS patterns calculated on A-type at $t=0$ and $\bar{X} / \bar{L}=0.1$.

contour line is adopted to distinguish the relative magnitude between 100 and 0.1 . This tendency would be expected from the comparison between the distributions shown in Figures 4(a) and 5(a).

Figure 10 shows the $\mu$-dependence of the scattered intensity distribution calculated by using the same values of the parameters listed in Figure 9 except $\bar{X} / \bar{L} . \bar{X} / \bar{L}$ is fixed to be 0.1 in order to check the effect of lamellar length on the distribution. The lamellar length in this case is shorter than that in Figure 9. Com-

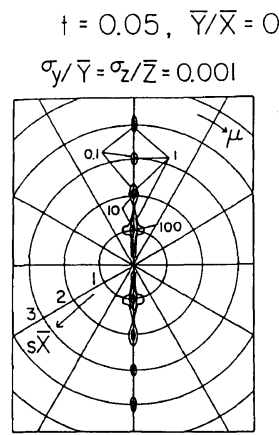

(a) $\sigma_{\alpha}=100$

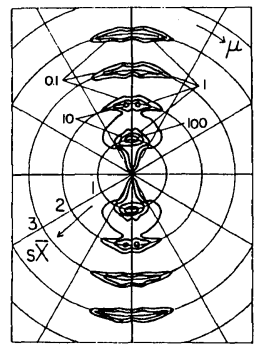

(b) $\sigma_{\alpha}=5$

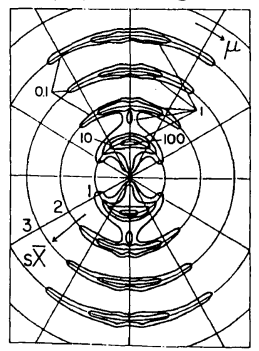

(c) $\sigma_{\alpha}=3$

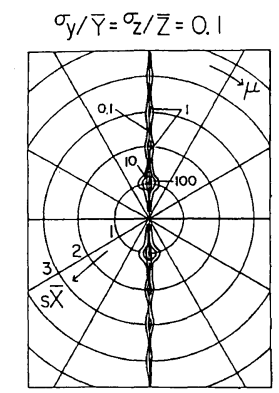

(d) $\sigma_{\alpha}=100$

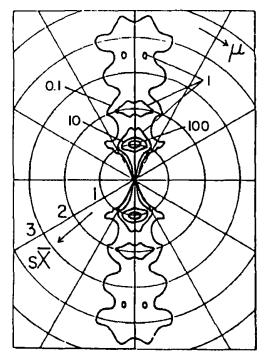

(e) $\sigma_{\alpha}=5$

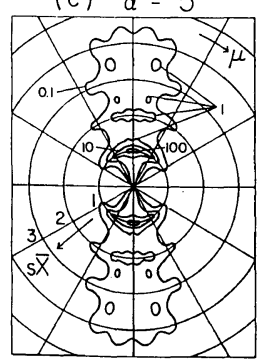

(f) $\sigma_{\alpha}=3$
Figure 11. SAXS patterns calculated on A-type at $t / \bar{X}=0.05$ and $\bar{X} / \bar{L}=0.02$.

paring the results in Figure 10 with those in Figure 9, the first and second order intensity distributions for the $\mu$-dependence become broader as lemellar length becomes smaller. Furthermore the change in profile of the distribution with variation of $\sigma_{y} / \bar{Y}, \sigma_{z} / Z$, and $\sigma_{\alpha}$ exhibits the similar tendency to the results in Figure 9. Namely, the increase in $\sigma_{y} / \bar{Y}$ and $\sigma_{z} / \bar{Z}$ makes the third and fourth order peak intensity distributions indistinct and this tendency becomes considerable when the distribution $p(\alpha)$ becomes broader.

Figure 11 shows the patterns calculated to understand the effect of the transition zones $t$ 
and $t^{\prime}$ on the $\mu$-dependence of the scattered intensity distribution. $t / \bar{X}$ is fixed as 0.05 . Furthermore $\bar{X} / \bar{L}$ is fixed to be 0.02 as listed in Figure 9. The change in profile of the patterns with variation of $\sigma_{y} / \bar{Y}, \sigma_{z} / \bar{Z}$, and $\sigma_{\alpha}$ shows similar tendency to that shown in Figure 9 except that the arc length representing the profile of the intensity distribution of the third and fourth order peaks is shorter than that in Figure 9. Such a phenomenon is due to the rapid decrease of the scattered intensity with increasing $s \bar{X}$, as $\mu$ increases. This has been a characteristics of the introduction of $t / \bar{X}^{10}$ The patterns (b) and (e) shown in Figures 10 and 11 are in good agreement with the intensity distribution observed for a sedimented mat of single crystals and also dry gel films of polyethylene. $^{8-10}$ Therefore the parameters $\bar{X} / \bar{L}$ and $t / \bar{X}$ are important to give the best fit between observed and calculated patterns. Unfortunately, it is very difficult to obtain the detailed information to determine those values experimentally on the basis of the observed SAXS patterns.

As can be seen in Figures 9-11, it should be noted that there are no many scattering maxima in the horizontal direction which appear in the patterns calculated on B-type model, namely, Blundell's model (see Figures 17 and 18 in ref 10 ). In order to seek the origin of this discrepancy, we consider the behavior of the intensity $I$ at $\mu=90^{\circ}$. For mathematical convenience, the treatment was restricted to the system at $\alpha=0^{\circ}$. This system denotes perfect orientation of the lamellar assemblies perpendicular to the $X_{3}$ axis, which is similar to the system given by $\sigma_{\alpha}=100$. At $\mu=90^{\circ}$, the scattered intensity $I$ derived from B-type model can be written as follows:

$I=\sum_{j=1}^{N} \sum_{k=1}^{N} f_{\mathrm{j}} f_{k}^{*}=N^{2}\left(\rho_{\mathrm{c}}-\rho_{\mathrm{a}}\right)(\bar{Y}-t) \frac{\sin ^{2}(\pi s \bar{L})}{(\pi s)^{2}}$

The term $\sin ^{2}(\pi s \bar{L})$ is a periodic function with maxima occuring at positions defined by

$$
\pi s \bar{L}=(2 n-1) \pi / 2 \quad(n, \text { integer })
$$

which can be rewritten as

$$
\frac{\bar{L}}{\bar{X}}=\frac{(2 n-1)}{2 s \bar{X}}
$$

Equation 53 shows that $\bar{L} / \bar{X}$ increases as $n$ increases and $s \bar{X}$ decreases. This indicates that the scattered intensity calculated from B-type has many scattering maxima in the horizontal direction.

On the other hand, at $\mu=90^{\circ}$ the scatted intensity calculated from A-type can be given by rewriting equations $7 \mathrm{a}-7 \mathrm{~d}$ follows:

$$
\begin{aligned}
& I_{1}=\sum_{j=1}^{N} \sum_{k=1}^{N} f_{j} f_{k}^{*}=N^{2}\left(\rho_{\mathrm{c}}-\bar{\rho}\right)^{2}(\bar{Y}-t)^{2} \frac{\sin ^{2}(\pi s \bar{L})}{(\pi s)^{2}} \\
& I_{2}=\sum_{j=1}^{N} \sum_{k=1}^{N} g_{j} g_{k}^{*}=N^{2}\left(\rho_{\mathrm{c}}-\bar{\rho}\right)^{2}\left(\bar{Z}-t^{\prime}\right)^{2} \frac{\sin ^{2}(\pi s \bar{L})}{(\pi s)^{2}} \\
& I_{3}=\sum_{j=1}^{N} \sum_{k=1}^{N} f_{j} g_{k}^{*}=N^{2}\left(\rho_{\mathrm{c}}-\bar{\rho}\right)\left(\rho_{\mathrm{a}}-\bar{\rho}\right)(\bar{Y}-t)\left(\bar{Z}-t^{\prime}\right) \frac{\sin ^{2}(\pi s \bar{L})}{(\pi s)^{2}}
\end{aligned}
$$

and

$$
I_{4}=\sum_{j=1}^{N} \sum_{k=1}^{N} g_{j} f_{j}^{*}=N^{2}\left(\rho_{\mathrm{c}}-\bar{\rho}\right)\left(\rho_{\mathrm{a}}-\bar{\rho}\right)(\bar{Y}-t)\left(\bar{Z}-t^{\prime}\right) \frac{\sin ^{2}(\pi s \bar{L})}{(\pi s)^{2}}
$$

Thus, 


$$
\begin{aligned}
I & =I_{1}+I_{2}+I_{3}+I_{4} \\
& =N^{2}\left\{\left(\rho_{\mathrm{c}}-\bar{\rho}\right)(\bar{Y}-t)-\left(\bar{\rho}-\rho_{\mathrm{a}}\right)\left(\bar{Z}-t^{\prime}\right)\right\}^{2} \frac{\sin ^{2}(\pi s \bar{L})}{(\pi s)^{2}}=0
\end{aligned}
$$

The zero value of eq 55 can be demonstrated from eq 18. From this result, it would be expected that the scattered intensity at $\mu=90^{\circ}$ takes a very small value when the lamellar assemblies have a sharp distribution with respect to the $X_{3}$ axis. This result is very reasonable, because scattering maxima do not appear in the horizontal direction. This indicates that A-type is better than B-type to describe the SAXS intensity distribution quantitatively. Therefore, it can be concluded that calculations of intensity must be carried out on the basis of the concept that scattering is due to positive and negative variations from the average density of the system.

Finally, we will consider the importance of the $I_{\mathrm{C}}$ term which has been sometimes neglected. According to the concepts proposed by Hosemann $^{4,5}$ and Blundell, ${ }^{6,7}$ the normalized scattered intensity must be zero when there is no fluctuation in the lamellar thickness and $N$ is infinite. The concept is obviously incorrect. This invokes the importance of eq 42 and 43 . In eq 43 , the term $\sin ^{2}(\pi N b \bar{X}) / \sin ^{2}(\pi b \bar{X})$, which is well-known as the Laue function, is included in the $I_{\mathrm{C}}$ term. The Laue function has the value $N^{2}$ at $s \bar{X}=n$ ( $n$, integer). This indicates that the scattered intensity $I$ cannot be approximated by the $I_{\mathrm{B}}$ term except when the lamellar thickness fluctuates and $N$ is sufficiently large. Therefore, the scattered intensity $I$ must be defined as a summation of the $I_{\mathrm{B}}$ and $I_{\mathrm{C}}$ terms. A similar result was already obtained for the one-dimensional problem. ${ }^{3}$

\section{CONCLUSION}

It is very important to introduce the concept that small angle scattering is due to positive and negative density deviation from the average density of the system. This can be de- monstrated obviously by considering the azimuthal angle $\mu$-dependence of the scattered intensity distribution. When the theoretical calculation was carried out using the above concept, namely, A-type, the pattern is in good agreement with one which has been observed usually for a sedimented mat of single crystal and dry gel films of polyethylene. ${ }^{8-10}$ By contrast, when the pattern was calculated on the basis of the concept that the scattering depends upon the excess electron density between the crystal and amorphous regions shown as B-type in Figure 3, the pattern has many peaks in the horizontal direction which have never been observed. This discrepancy could be solved mathematically through eq $51-55$. However, as shown in Figures 4-6, the intensity distributions at $\mu=0^{\circ}$ calculated from A-type and B-type resemble each other except on the region of $0 \leqq s \bar{X} \leqq 1$. Therefore, It can concluded that the azimuthal angle $\mu$ dependence is very important to emphasize the validity of A-type.

Acknowledgement. We thank to Dr. Masaki Tsuji of Kyoto University, Chemical Research Institute, Uji, Japan.

\section{APPENDIX}

In the previous paper, ${ }^{3}$ it can be demonstrated that Babinet's theorem is satisfied for crystallinity (in the case of a semicrystalline polymer) or volume fraction (for a block copolymer), when there is no fluctuation in the thickness of the alternating lamellar phases in one dimension. Now, let us consider whether Babinet's theorem is satisfied for $X_{\mathrm{c}}$ in eq 43 representing the scattered intensity from the oriented lamellar phases in the two dimensional system. For this purpose, we shall re- 
turn to eq 21 . From eq 21 we have

$$
p=X_{\mathrm{c}}+\left(\frac{1-2 X_{\mathrm{c}}}{1-X_{\mathrm{c}}}\right) \frac{t}{\bar{X}}
$$

When $X_{\mathrm{c}}^{\prime}$ is employed instead of $1-X_{\mathrm{c}}$, eq A1 can be rewritten as:

$$
p=1-X_{\mathrm{c}}^{\prime}+\left(\frac{2 X_{\mathrm{c}}^{\prime}-1}{X_{\mathrm{c}}^{\prime}}\right) \frac{t}{\bar{X}}
$$

By using the relation $t^{\prime}=X_{\mathrm{c}} t /\left(1-X_{\mathrm{c}}\right)=$ $\left(1-X_{\mathrm{c}}^{\prime}\right) t / X_{\mathrm{c}}^{\prime}$ which can be derived from eq 21 and 22 we have

$$
p=1-\left\{X_{\mathrm{c}}^{\prime}+\left(\frac{1-2 X_{\mathrm{c}}^{\prime}}{1-X_{\mathrm{c}}^{\prime}}\right) \frac{t^{\prime}}{\bar{X}}\right\}=1-p^{\prime}
$$

Therefore, we can substitute $1-X_{\mathrm{c}}^{\prime}$ and $1-p^{\prime}$ into eq 43 instead of $X_{\mathrm{c}}$ and $p$, respectively. Thus we have

$$
\begin{aligned}
I / N \bar{L} \bar{X}= & -I_{\mathrm{C}} \\
= & \int_{0}^{2 \pi} p(\alpha)\left\{\frac{\left(\rho_{\mathrm{c}}-\rho_{\mathrm{a}}\right)\left(1-2 X_{\mathrm{c}}^{\prime}\right) \sin \pi a \bar{L}}{(\pi b)^{2}\left(p-X_{\mathrm{c}}^{\prime}\right) \bar{X} \pi a}\right\}^{2} \frac{\sin ^{2}(\pi N b \bar{X})}{N \bar{X} \bar{L} \sin ^{2}(\pi b \bar{X})} \\
& \times\left\{\sin ^{2}\left[\pi b\left(1-X_{\mathrm{c}}^{\prime}\right)\left(p^{\prime}-X_{\mathrm{c}}^{\prime}\right) \bar{X} /\left(1-2 X_{\mathrm{c}}^{\prime}\right)\right] \sin ^{2}\left[\pi b X_{\mathrm{c}}^{\prime}\left(1-X_{\mathrm{c}}^{\prime}-p^{\prime}\right) \bar{X} /\left(1-2 X_{\mathrm{c}}^{\prime}\right)\right]\right. \\
& +\sin ^{2}\left[\pi b X_{\mathrm{c}}^{\prime}\left(p^{\prime}-X_{\mathrm{c}}^{\prime}\right) \bar{X} /\left(1-2 X_{\mathrm{c}}^{\prime}\right)\right] \sin ^{2}\left[\pi b\left(1-X_{\mathrm{c}}^{\prime}\right)\left(1-X_{\mathrm{c}}^{\prime}-p^{\prime}\right) \bar{X} /\left(1-2 X_{\mathrm{c}}^{\prime}\right)\right] \\
- & 2 \sin \left[\pi b\left(1-X_{\mathrm{c}}^{\prime}\right)\left(p^{\prime}-X_{\mathrm{c}}^{\prime}\right) \bar{X} /\left(1-2 X_{\mathrm{c}}^{\prime}\right)\right] \sin \left[\pi b X_{\mathrm{c}}^{\prime}\left(1-X_{\mathrm{c}}^{\prime}-p^{\prime}\right) \bar{X} /\left(1-2 X_{\mathrm{c}}^{\prime}\right)\right] \\
& \times \sin \left[\pi b X_{\mathrm{c}}^{\prime}\left(p^{\prime}-X_{\mathrm{c}}^{\prime}\right) \bar{X} /\left(1-2 X_{\mathrm{c}}^{\prime}\right)\right] \sin \left[\pi b\left(1-X_{\mathrm{c}}^{\prime}\right)\left(1-X_{\mathrm{c}}^{\prime}-p^{\prime}\right) \bar{X} /\left(1-2 X_{\mathrm{c}}^{\prime}\right)\right] \\
& \times \cos (\pi b \bar{X})\} \mathrm{d} \alpha
\end{aligned}
$$

Comparison of eq 43 and A4 indicates that Babinet's theorem is satisfied for $X_{\mathrm{c}}$ when the two phases have no fluctuation in thickness as discussed in the previous paper. ${ }^{3}$ However, Babinet's theorem is not satisfied in the $I_{\mathrm{C}}$ term as derived by Hashimoto et al. ${ }^{11}$ This indicates that $I_{2}^{\mathrm{C}}$ in eq 27 (or eq 40) and $I_{3}^{\mathrm{C}}$ in eq 29 (or eq 41) are very important for representing the scattered intensity in a real system.

Incidentally, eq 44 for the system without transition zones satisfies Babinet's theorem for $X_{\mathrm{c}}$, but eq 45 derived from Model B corresponding to eq 44 does not satisfy it.

\section{REFERENCES}

1. W. Wenig, and R. Brämer, Colloid Polym. Sci., 256, 125 (1978).

2. H. Meyer and H.-G. Killian, Progr. Colloid Polym. Sci., 64, 154 (1978).
3. M. Matsuo, C. Sawatari, M. Tsuji, and R. S. T. Manley, J. Chem. Soc., Faraday Trans. 2, 79, 1593 (1983).

4. R. Hosemann, Z. Phys., 127, 16 (1949).

5. R. Hosemann and S. N. Bagchi, "Direct Analysis of Diffraction by Matter," Amsterdam, North Holland, 1962.

6. D. J. Blundell, Acta Cryst., A26, 472 (1970).

7. D. J. Blundell, Acta Cryst., A26, 476 (1970).

8. M. Matsuo and R. S. T. Manley, Macromolecules, 15, 985 (1982).

9. M. Matsuo and R. S. T. Manley, Macromolecules, 16, 1500 (1983).

10. M. Matsuo, M. Tsuji, and R. S. T. Manley, Macromolecules, 16, 1505 (1983).

11. T. Hashimoto, K. Nagatoshi, A. Todo, $\mathrm{H}$. Hasegawa, and H. Kawai, Macromolecules, 7, 264 (1974).

12. M. Matsuo, M. Yoneda, and M. Iida, Macromolecules, submitted.

13. P. Smith and P. J. Lemstra, J. Mater. Sci., 15, 505 (1980). 